\title{
Comparison of Classical Method, Extension Principle and $\alpha$-Cuts and Interval Arithmetic Method in Solving System of Fuzzy Linear Equations
}

\author{
Sahidul Islam1, Md. Saiduzzaman'1, Md. Shafiqul Islam¹, Abeda Sultana² \\ ${ }^{1}$ Department of Mathematics, IUBAT (International University of Business Agriculture and Technology), Dhaka, Bangladesh \\ ${ }^{2}$ Department of Mathematics, Jahangirnagar University, Dhaka, Bangladesh \\ Email: sahidul.sohag@iubat.edu, szaman@iubat.edu, shafiqul.islam@iubat.edu, abedamathju@yahoo.co.uk
}

How to cite this paper: Islam, S., Saiduzzaman, Md., Islam, Md.S. and Sultana, A. (2019) Comparison of Classical Method, Extension Principle and $\alpha$-Cuts and Interval Arithmetic Method in Solving System of Fuzzy Linear Equations. American Journal of Computational Mathematics, 9, 1-24. https://doi.org/10.4236/ajcm.2019.91001

Received: December 10, 2018

Accepted: February 12, 2019

Published: February 15, 2019

Copyright $\odot 2019$ by author(s) and Scientific Research Publishing Inc. This work is licensed under the Creative Commons Attribution-NonCommercial International License (CC BY-NC 4.0).

http://creativecommons.org/licenses/by-nc/4.0/ (c) (i) (8) Open Access

\begin{abstract}
The system of linear equations plays a vital role in real life problems such as optimization, economics, and engineering. The parameters of the system of linear equations are modeled by taking the experimental or observation data. So the parameters of the system actually contain uncertainty rather than the crisp one. The uncertainties may be considered in term of interval or fuzzy numbers. In this paper, a detailed study of three solution techniques namely Classical Method, Extension Principle method and $\alpha$-cuts and interval Arithmetic Method to solve the system of fuzzy linear equations has been done. Appropriate applications are given to illustrate each technique. Then we discuss the comparison of the different methods numerically and graphically.
\end{abstract}

\section{Keywords}

Fuzzy Set, Classical Solution, Extension Principle,

$\alpha$-Cut and Interval Arithmetic Method

\section{Introduction}

There are many linear equation systems in many areas of science and engineering. According to Moore [1], exact numerical data might be unrealistic, but there could be considered uncertain data as more aspects of a real word problem. Fuzzy data are being used as a natural way to describe uncertain data. So, we need to solve those linear systems in which all parameters or some of them are fuzzy numbers. Friedman et al. [2] [3] applied an embedding method for solving 
$A x=b$, where $A$ is a nonsingular crisp matrix. There are many other numerical methods for solving fuzzy linear system such as Jacobi, Gauss-Seidel, Adomiam decomposition method and SOR iterative method [4] [5] [6] [7]. Dehgan in [8], [9] introduced the full fuzzy system in which $b$ and $A$ are fuzzy vector and fuzzy matrix, respectively. Then Kumar in [10] obtained an exact solution of fully fuzzy linear system by solving linear programming.

In 1965, Lotfi A. Zadeh [11], professor of electrical engineering at the University of California (Berkley), published the first of his paper on his new theory of Fuzzy Sets and System. After the development of fuzzy set theory, researchers have successfully applied this in economics. Buckley [12] applied fuzzy mathematics in finance; in 1992 Buckley devised a technique to solve fuzzy equations in economics and finance. The above methods made us inspired to work on the solution techniques and finally, we got something. The objective of this paper is to present three different and effective methods to solve the system of fuzzy linear equation. Furthermore, we show the comparison among the methods with the help of numerical calculation as well as graphical representations. The paper organized as follows. In Section 2 we set some basic definitions and notation list. Section 3 deals with the methods. The applications of the models are presented in Section 4 and finally, Section 5 shows the results of the models.

\section{Notations and Definitions}

\subsection{Notations List}

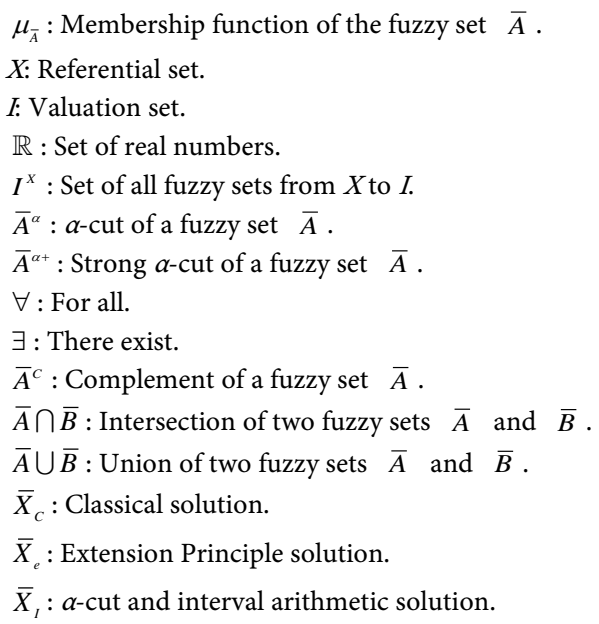

\subsection{Fuzzy Sets}

A fuzzy set [3] is a class of objects with a continuum of the grade of membership. Let $X$ be a space of points. A fuzzy set $A$ in $X$ is characterized by a membership function which associates with each points in $X$ a real number $\mu_{A}(x)$ in the interval $[0,1]$ with the value of $\mu_{A}$ at $X$ representing the grade of membership of $x$ in $A$. Thus the nearer to the value of $\mu_{A}$ to unity, the higher the grade of membership of $x$ in $A$. 


\subsection{Fuzzy Linear Equation}

Fuzzy linear equations are similar to ordinary linear equations in classical mathematics. A fuzzy linear equation is of the form $\bar{A} \cdot \bar{X}=\bar{B}$ or $\bar{A}+\bar{X}=\bar{B}$ or $\bar{A} \cdot \bar{X}+\bar{C}=\bar{B}$ where $\bar{A}, \bar{B}$ and $\bar{C}$ are given fuzzy numbers and $\bar{X}$ is an unknown fuzzy number by which the equation is satisfied.

\subsection{The System of Fuzzy Linear Equations}

A system of fuzzy linear equations is of the form $\bar{A} \cdot \bar{X}=\bar{B}$, where $\bar{A}=\left[\bar{a}_{i j}\right]$ is a $n \times n$ matrix of fuzzy numbers $\bar{a}_{i j}, \bar{X}^{t}=\left(\bar{x}_{1}, \bar{x}_{2}, \cdots, \bar{x}_{n}\right)$ is an unknown $n \times 1$ vector of fuzzy numbers $\bar{x}_{i}$ by which the equation is satisfied and $\bar{B}^{t}=\left(\bar{b}_{1}, \bar{b}_{2}, \cdots, \bar{b}_{n}\right)$ is a $n \times 1$ vector of fuzzy numbers $\bar{b}_{i}$. Now we can write corresponding $n \times n$ system for all $\bar{a}_{i j} \in \mathbb{R}, 1 \leq i, j \leq n$ as follows:

$$
\left(\begin{array}{cccc}
\bar{a}_{11} & \bar{a}_{11} & \cdots & \bar{a}_{11} \\
\bar{a}_{21} & \bar{a}_{22} & \cdots & \bar{a}_{2 n} \\
\vdots & \vdots & \ddots & \vdots \\
\bar{a}_{n 1} & \bar{a}_{n 2} & \cdots & \bar{a}_{n n}
\end{array}\right)\left(\begin{array}{c}
\bar{x}_{1} \\
\bar{x}_{2} \\
\vdots \\
\bar{x}_{n}
\end{array}\right)=\left(\begin{array}{c}
\bar{b}_{1} \\
\bar{b}_{2} \\
\vdots \\
\bar{b}_{n}
\end{array}\right)
$$

\section{Methods}

\subsection{Classical Solution}

We denote the classical solution of $\bar{A} \cdot \bar{X}=\bar{B}$ as $\bar{X}_{c}$, if it exists.

Substitute the $\alpha$-cuts of $\bar{a}_{i j}, \bar{x}_{i}$ and $\bar{b}_{i}$ for $\bar{a}_{i j}, \bar{x}_{i}$ and $\bar{b}_{i} \quad(1 \leq i, j \leq 3)$ respectively in the system of linear equations

$$
\begin{aligned}
& \bar{a}_{11} \bar{x}_{1}+\bar{a}_{12} \bar{x}_{2}+\bar{a}_{13} \bar{x}_{3}=\bar{b}_{1}, \\
& \bar{a}_{21} \bar{x}_{1}+\bar{a}_{22} \bar{x}_{2}+\bar{a}_{23} \bar{x}_{3}=\bar{b}_{2}, \\
& \bar{a}_{31} \bar{x}_{1}+\bar{a}_{32} \bar{x}_{2}+\bar{a}_{33} \bar{x}_{3}=\bar{b}_{3},
\end{aligned}
$$

After substituting the $\alpha$-cuts of $\bar{a}_{i j}, \bar{x}_{i}$ and $\bar{b}_{i}$ for $\bar{a}_{i j}, \bar{x}_{i}$ and $\bar{b}_{i}$ $(1 \leq i, j \leq 3)$ in the Equations (1)-(3), we get the following three interval equations $\forall \alpha \in[0,1]$,

$$
\begin{aligned}
& {\left[a_{11 L}(\alpha), a_{11 U}(\alpha)\right] \cdot\left[x_{1 L}(\alpha), x_{1 U}(\alpha)\right]+\left[a_{12 L}(\alpha), a_{12 U}(\alpha)\right] \cdot\left[x_{2 L}(\alpha), x_{2 U}(\alpha)\right]} \\
& +\left[a_{13 L}(\alpha), a_{13 U}(\alpha)\right] \cdot\left[x_{3 L}(\alpha), x_{3 U}(\alpha)\right]=\left[b_{1 L}(\alpha), b_{1 U}(\alpha)\right] \\
& {\left[a_{21 L}(\alpha), a_{21 U}(\alpha)\right] \cdot\left[x_{1 L}(\alpha), x_{1 U}(\alpha)\right]+\left[a_{22 L}(\alpha), a_{22 U}(\alpha)\right] \cdot\left[x_{2 L}(\alpha), x_{2 U}(\alpha)\right]} \\
& +\left[a_{23 L}(\alpha), a_{23 U}(\alpha)\right] \cdot\left[x_{3 L}(\alpha), x_{3 U}(\alpha)\right]=\left[b_{2 L}(\alpha), b_{2 U}(\alpha)\right] \\
& {\left[a_{31 L}(\alpha), a_{31 U}(\alpha)\right] \cdot\left[x_{1 L}(\alpha), x_{1 U}(\alpha)\right]+\left[a_{32 L}(\alpha), a_{32 U}(\alpha)\right] \cdot\left[x_{2 L}(\alpha), x_{2 U}(\alpha)\right](6)} \\
& +\left[a_{33 L}(\alpha), a_{33 U}(\alpha)\right] \cdot\left[x_{3 L}(\alpha), x_{3 U}(\alpha)\right]=\left[b_{3 L}(\alpha), b_{3 U}(\alpha)\right]
\end{aligned}
$$

We now need to simplify these equations.

Assuming that all the $\bar{a}_{i j}$ and $\bar{b}_{i}$ are triangular fuzzy numbers and put $\alpha=1$ in Equations (4)-(6). Then we obtain the crisp linear system of equations

$$
a_{11} x_{1}+a_{12} x_{2}+a_{13} x_{3}=b_{1}
$$




$$
\begin{aligned}
& \qquad \begin{array}{l}
a_{21} x_{1}+a_{22} x_{2}+a_{23} x_{3}=b_{2} \\
a_{31} x_{1}+a_{32} x_{2}+a_{33} x_{3}=b_{3}
\end{array} \\
& \text { The sign of the solutions } x_{1}, x_{2} \text { and } x_{3} \text { determines the sign of the unknown } \\
& \text { fuzzy numbers } \bar{x}_{1}, \bar{x}_{2} \text { and } \bar{x}_{3} \text {. Let us assume for this discussion that all the } \\
& \bar{a}_{i j}>0 \text { and all the } \bar{b}_{i}>0 \text {, so that we try for } \bar{x}_{i}>0, i=1,2,3 \text {. We get from Equ- } \\
& \text { ations }(4)-(6) \\
& \left.\qquad a_{11 L}(\alpha) \cdot x_{1 L}(\alpha), a_{11 U}(\alpha) \cdot x_{1 U}(\alpha)\right]+\left[a_{12 L}(\alpha) \cdot x_{2 L}(\alpha), a_{12 U}(\alpha) \cdot x_{2 U}(\alpha)\right] \\
& +\left[a_{13 L}(\alpha) \cdot x_{3 L}(\alpha), a_{13 U}(\alpha) \cdot x_{3 U}(\alpha)\right]=\left[b_{1 L}(\alpha), b_{1 U}(\alpha)\right] \\
& \quad\left[a_{21 L}(\alpha) \cdot x_{1 L}(\alpha), a_{21 U}(\alpha) \cdot x_{1 U}(\alpha)\right]+\left[a_{22 L}(\alpha) \cdot x_{2 L}(\alpha), a_{22 U}(\alpha) \cdot x_{2 U}(\alpha)\right] \\
& +\left[a_{23 L}(\alpha) \cdot x_{3 L}(\alpha), a_{23 U}(\alpha) \cdot x_{3 U}(\alpha)\right]=\left[b_{2 L}(\alpha), b_{2 U}(\alpha)\right] \\
& \quad\left[a_{31 L}(\alpha) \cdot x_{1 L}(\alpha), a_{31 U}(\alpha) \cdot x_{1 U}(\alpha)\right]+\left[a_{32 L}(\alpha) \cdot x_{2 L}(\alpha), a_{32 U}(\alpha) \cdot x_{2 U}(\alpha)\right] \\
& +\left[a_{33 L}(\alpha) \cdot x_{3 L}(\alpha), a_{33 U}(\alpha) \cdot x_{3 U}(\alpha)\right]=\left[b_{3 L}(\alpha), b_{3 U}(\alpha)\right]
\end{aligned}
$$

which yields a $6 \times 6$ crisp system of linear equations as below

$$
\begin{aligned}
& a_{11 L}(\alpha) \cdot x_{1 L}(\alpha)+a_{12 L}(\alpha) \cdot x_{2 L}(\alpha)+a_{13 L}(\alpha) \cdot x_{3 L}(\alpha)=b_{1 L}(\alpha) \\
& a_{21 L}(\alpha) \cdot x_{1 L}(\alpha)+a_{22 L}(\alpha) \cdot x_{2 L}(\alpha)+a_{23 L}(\alpha) \cdot x_{3 L}(\alpha)=b_{2 L}(\alpha) \\
& a_{31 L}(\alpha) \cdot x_{1 L}(\alpha)+a_{32 L}(\alpha) \cdot x_{2 L}(\alpha)+a_{33 L}(\alpha) \cdot x_{3 L}(\alpha)=b_{3 L}(\alpha) \\
& a_{11 U}(\alpha) \cdot x_{1 U}(\alpha)+a_{12 U}(\alpha) \cdot x_{2 U}(\alpha)+a_{13 U}(\alpha) \cdot x_{3 U}(\alpha)=b_{1 U}(\alpha) \\
& a_{21 U}(\alpha) \cdot x_{1 U}(\alpha)+a_{22 U}(\alpha) \cdot x_{2 U}(\alpha)+a_{23 U}(\alpha) \cdot x_{3 U}(\alpha)=b_{2 U}(\alpha) \\
& a_{31 U}(\alpha) \cdot x_{1 U}(\alpha)+a_{32 U}(\alpha) \cdot x_{2 U}(\alpha)+a_{33 U}(\alpha) \cdot x_{3 U}(\alpha)=b_{3 U}(\alpha)
\end{aligned}
$$

We solve this system for $x_{i L}(\alpha)$ and $x_{i U}(\alpha), i=1,2,3, \alpha \in[0,1]$.

Using matrix notation this system can be written as

$$
\left[\begin{array}{cccccc}
a_{11 L}(\alpha) & a_{12 L}(\alpha) & a_{13 L}(\alpha) & 0 & 0 & 0 \\
a_{21 L}(\alpha) & a_{22 L}(\alpha) & a_{23 L}(\alpha) & 0 & 0 & 0 \\
a_{31 L}(\alpha) & a_{32 L}(\alpha) & a_{33 L}(\alpha) & 0 & 0 & 0 \\
0 & 0 & 0 & a_{11 U}(\alpha) & a_{12 U}(\alpha) & a_{13 U}(\alpha) \\
0 & 0 & 0 & a_{21 U}(\alpha) & a_{22 U}(\alpha) & a_{23 U}(\alpha) \\
0 & 0 & 0 & a_{31 U}(\alpha) & a_{32 U}(\alpha) & a_{33 U}(\alpha)
\end{array}\right] \cdot\left[\begin{array}{c}
x_{1 L}(\alpha) \\
x_{2 L}(\alpha) \\
x_{3 L}(\alpha) \\
x_{1 U}(\alpha) \\
x_{2 U}(\alpha) \\
x_{3 U}(\alpha)
\end{array}\right]=\left[\begin{array}{c}
b_{1 L}(\alpha) \\
b_{2 L}(\alpha) \\
b_{3 L}(\alpha) \\
b_{1 U}(\alpha) \\
b_{2 U}(\alpha) \\
b_{3 U}(\alpha)
\end{array}\right]
$$

Using

$$
W=\left[\begin{array}{cccccc}
a_{11 L}(\alpha) & a_{12 L}(\alpha) & a_{13 L}(\alpha) & 0 & 0 & 0 \\
a_{21 L}(\alpha) & a_{22 L}(\alpha) & a_{23 L}(\alpha) & 0 & 0 & 0 \\
a_{31 L}(\alpha) & a_{32 L}(\alpha) & a_{33 L}(\alpha) & 0 & 0 & 0 \\
0 & 0 & 0 & a_{11 U}(\alpha) & a_{12 U}(\alpha) & a_{13 U}(\alpha) \\
0 & 0 & 0 & a_{21 U}(\alpha) & a_{22 U}(\alpha) & a_{23 U}(\alpha) \\
0 & 0 & 0 & a_{31 U}(\alpha) & a_{32 U}(\alpha) & a_{33 U}(\alpha)
\end{array}\right], \quad S=\left[\begin{array}{c}
x_{1 L}(\alpha) \\
x_{2 L}(\alpha) \\
x_{3 L}(\alpha) \\
x_{1 U}(\alpha) \\
x_{2 U}(\alpha) \\
x_{3 U}(\alpha)
\end{array}\right] \text { and } V=\left[\begin{array}{c}
b_{1 L}(\alpha) \\
b_{2 L}(\alpha) \\
b_{3 L}(\alpha) \\
b_{1 U}(\alpha) \\
b_{2 U}(\alpha) \\
b_{3 U}(\alpha)
\end{array}\right]
$$

we get a crisp system of the form $W \cdot S=V$.

For obtaining the fuzzy solution for the fully fuzzy linear system of equations, the necessary condition is that the coefficient matrix of the converted crisp sys- 
tem is invertible $\forall \alpha \in[0,1]$.

The system (5.2.29) can be partitioned into two system as

$$
\left[\begin{array}{lll}
a_{11 L}(\alpha) & a_{12 L}(\alpha) & a_{13 L}(\alpha) \\
a_{21 L}(\alpha) & a_{22 L}(\alpha) & a_{23 L}(\alpha) \\
a_{31 L}(\alpha) & a_{32 L}(\alpha) & a_{33 L}(\alpha)
\end{array}\right] \cdot\left[\begin{array}{c}
x_{1 L}(\alpha) \\
x_{2 L}(\alpha) \\
x_{3 L}(\alpha)
\end{array}\right]=\left[\begin{array}{l}
b_{1 L}(\alpha) \\
b_{2 L}(\alpha) \\
b_{3 L}(\alpha)
\end{array}\right]
$$

and

$$
\left[\begin{array}{lll}
a_{11 U}(\alpha) & a_{12 U}(\alpha) & a_{13 U}(\alpha) \\
a_{21 U}(\alpha) & a_{22 U}(\alpha) & a_{23 U}(\alpha) \\
a_{31 U}(\alpha) & a_{32 U}(\alpha) & a_{33 U}(\alpha)
\end{array}\right] \cdot\left[\begin{array}{c}
x_{1 U}(\alpha) \\
x_{2 U}(\alpha) \\
x_{3 U}(\alpha)
\end{array}\right]=\left[\begin{array}{l}
b_{1 U}(\alpha) \\
b_{2 U}(\alpha) \\
b_{3 U}(\alpha)
\end{array}\right]
$$

That is, $W_{L} \cdot S_{L}=V_{L}$ and $W_{U} \cdot S_{U}=V_{U}$. The solution of these crisp systems determines $x_{i L}(\alpha)$ and $x_{i U}(\alpha), i=1,2,3, \alpha \in[0,1]$, which are used to reconstruct the components of $3 \times 1$ fuzzy vector $\bar{X}$.

After solving for the $x_{i L}(\alpha)$ and $x_{i U}(\alpha), i=1,2,3, \alpha \in[0,1]$ we check to see if the intervals $\bar{x}_{i}=\left[x_{i L}(\alpha), x_{i U}(\alpha)\right], i=1,2,3, \alpha \in[0,1]$ define continuous fuzzy numbers for $i=1,2$. What is needed is:

1) $\frac{\partial}{\partial \alpha}\left(x_{i L}(\alpha)\right)>0$,

2) $\frac{\partial}{\partial \alpha}\left(x_{i U}(\alpha)\right)<0$, and

3) $x_{i L}(1) \leq x_{i U}(1)$ for $i=1,2,3$ (equality for triangular shaped fuzzy numbers).

\subsection{Extension Principle Solution}

We denote the extension principle solution of a system of fuzzy linear equations by $\bar{X}_{e}$ and it always exists but may, or may not satisfy the original system of fuzzy linear equations. That is, $\bar{A} \cdot \bar{X}_{e}=\bar{B}$ may, or may not be true.

Let the components of $\bar{X}_{e}$ are $\bar{X}_{1}, \bar{X}_{2}$ and $\bar{X}_{3}$. In this method, we need to fuzzify the crisp solutions

$$
\begin{aligned}
& x_{1}=\frac{b_{1}\left(a_{22} a_{33}-a_{23} a_{32}\right)-a_{12}\left(a_{33} b_{2}-a_{23} b_{3}\right)+a_{13}\left(a_{32} b_{2}-a_{22} b_{3}\right)}{a_{11}\left(a_{22} a_{33}-a_{32} a_{23}\right)-a_{12}\left(a_{21} a_{33}-a_{31} a_{23}\right)+a_{13}\left(a_{21} a_{32}-a_{31} a_{22}\right)} \\
& x_{2}=\frac{a_{11}\left(a_{33} b_{2}-a_{23} b_{3}\right)-b_{1}\left(a_{21} a_{33}-a_{31} a_{23}\right)+a_{13}\left(a_{21} b_{3}-a_{31} b_{2}\right)}{a_{11}\left(a_{22} a_{33}-a_{32} a_{23}\right)-a_{12}\left(a_{21} a_{33}-a_{31} a_{23}\right)+a_{13}\left(a_{21} a_{32}-a_{31} a_{22}\right)} \\
& x_{3}=\frac{a_{11}\left(a_{22} b_{3}-a_{32} b_{2}\right)-a_{12}\left(a_{21} b_{3}-a_{31} b_{2}\right)+b_{1}\left(a_{21} a_{32}-a_{31} a_{22}\right)}{a_{11}\left(a_{22} a_{33}-a_{32} a_{23}\right)-a_{12}\left(a_{21} a_{33}-a_{31} a_{23}\right)+a_{13}\left(a_{21} a_{32}-a_{31} a_{22}\right)}
\end{aligned}
$$

using the extension principle.

Let

$$
\begin{aligned}
& h_{1}\left(a_{11}, a_{12}, a_{13}, a_{21}, a_{22}, a_{23}, a_{31}, a_{32}, a_{33}, b_{1}, b_{2}, b_{3}\right)=x_{1}, \\
& h_{2}\left(a_{11}, a_{12}, a_{13}, a_{21}, a_{22}, a_{23}, a_{31}, a_{32}, a_{33}, b_{1}, b_{2}, b_{3}\right)=x_{2},
\end{aligned}
$$

and 


$$
h_{3}\left(a_{11}, a_{12}, a_{13}, a_{21}, a_{22}, a_{23}, a_{31}, a_{32}, a_{33}, b_{1}, b_{2}, b_{3}\right)=x_{3} .
$$

To obtain the first component $\bar{x}_{1}$ in $\bar{X}_{e}$, we substitute

$$
\bar{a}_{11}, \bar{a}_{12}, \bar{a}_{13}, \bar{a}_{21}, \bar{a}_{22}, \bar{a}_{23}, \bar{a}_{31}, \bar{a}_{32}, \bar{a}_{33}, \bar{b}_{1}, \bar{b}_{2}, \bar{b}_{3}
$$

for

$$
a_{11}, a_{12}, a_{13}, a_{21}, a_{22}, a_{23}, a_{31}, a_{32}, a_{33}, b_{1}, b_{2}, b_{3}
$$

in $h_{1}\left(a_{11}, a_{12}, a_{13}, a_{21}, a_{22}, a_{23}, a_{31}, a_{32}, a_{33}, b_{1}, b_{2}, b_{3}\right)$ and evaluate using the extension principle.

It should be noted that,

$$
\Delta=a_{11}\left(a_{22} a_{33}-a_{32} a_{23}\right)-a_{12}\left(a_{21} a_{33}-a_{31} a_{23}\right)+a_{13}\left(a_{21} a_{32}-a_{31} a_{22}\right) \neq 0,
$$

that is, the determinant of the coefficients matrix must be nonsingular and invertible.

Let $\alpha$-cut of $\bar{x}_{1}$ is $\bar{x}_{1}[\alpha]=\left[x_{1 L}(\alpha), x_{1 U}(\alpha)\right]$.

Then the $\alpha$-cut of $\bar{x}_{1}$ can be written as

$$
\begin{gathered}
x_{1 L}(\alpha)=\min \left\{h_{1}\left(a_{11}, a_{12}, a_{13}, a_{21}, a_{22}, a_{23}, a_{31}, a_{32}, a_{33}, b_{1}, b_{2}, b_{3}\right):\right. \\
\left.a_{i j} \in \bar{a}_{i j}[\alpha], b_{j} \in \bar{b}_{j}[\alpha]\right\} \\
x_{1 U}(\alpha)=\max \left\{h_{1}\left(a_{11}, a_{12}, a_{13}, a_{21}, a_{22}, a_{23}, a_{31}, a_{32}, a_{33}, b_{1}, b_{2}, b_{3}\right):\right. \\
\left.a_{i j} \in \bar{a}_{i j}[\alpha], b_{j} \in \bar{b}_{j}[\alpha]\right\}
\end{gathered}
$$

Or,

$$
\begin{gathered}
x_{1 L}(\alpha)=\min \left\{\frac{b_{1}\left(a_{22} a_{33}-a_{23} a_{32}\right)-a_{12}\left(a_{33} b_{2}-a_{23} b_{3}\right)+a_{13}\left(a_{32} b_{2}-a_{22} b_{3}\right)}{a_{11}\left(a_{22} a_{33}-a_{32} a_{23}\right)-a_{12}\left(a_{21} a_{33}-a_{31} a_{23}\right)+a_{13}\left(a_{21} a_{32}-a_{31} a_{22}\right)} ;\right. \\
\left.a_{i j} \in \bar{a}_{i j}[\alpha], b_{j} \in \bar{b}_{j}[\alpha]\right\} \\
x_{1 U}(\alpha)=\max \left\{\frac{b_{1}\left(a_{22} a_{33}-a_{23} a_{32}\right)-a_{12}\left(a_{33} b_{2}-a_{23} b_{3}\right)+a_{13}\left(a_{32} b_{2}-a_{22} b_{3}\right)}{a_{11}\left(a_{22} a_{33}-a_{32} a_{23}\right)-a_{12}\left(a_{21} a_{33}-a_{31} a_{23}\right)+a_{13}\left(a_{21} a_{32}-a_{31} a_{22}\right)} ;\right. \\
\left.a_{i j} \in \bar{a}_{i j}[\alpha], b_{j} \in \bar{b}_{j}[\alpha]\right\}
\end{gathered}
$$

for $\alpha \in[0,1]$ and $1 \leq i, j \leq 3$.

Similarly, $\alpha$-cut of $\bar{x}_{2}$ is $\bar{x}_{2}[\alpha]=\left[x_{2 L}(\alpha), x_{2 U}(\alpha)\right]$. Then the $\alpha$-cut of $\bar{x}_{2}$ is

$$
\begin{gathered}
x_{2 L}(\alpha)=\min \left\{\frac{a_{11}\left(a_{33} b_{2}-a_{23} b_{3}\right)-b_{1}\left(a_{21} a_{33}-a_{31} a_{23}\right)+a_{13}\left(a_{21} b_{3}-a_{31} b_{2}\right)}{a_{11}\left(a_{22} a_{33}-a_{32} a_{23}\right)-a_{12}\left(a_{21} a_{33}-a_{31} a_{23}\right)+a_{13}\left(a_{21} a_{32}-a_{31} a_{22}\right)} ;\right. \\
\left.a_{i j} \in \bar{a}_{i j}[\alpha], b_{j} \in \bar{b}_{j}[\alpha]\right\} \\
x_{2 U}(\alpha)=\max \left\{\frac{a_{11}\left(a_{33} b_{2}-a_{23} b_{3}\right)-b_{1}\left(a_{21} a_{33}-a_{31} a_{23}\right)+a_{13}\left(a_{21} b_{3}-a_{31} b_{2}\right)}{a_{11}\left(a_{22} a_{33}-a_{32} a_{23}\right)-a_{12}\left(a_{21} a_{33}-a_{31} a_{23}\right)+a_{13}\left(a_{21} a_{32}-a_{31} a_{22}\right)} ;\right. \\
\left.a_{i j} \in \bar{a}_{i j}[\alpha], b_{j} \in \bar{b}_{j}[\alpha]\right\}
\end{gathered}
$$


for $\alpha \in[0,1]$ and $1 \leq i, j \leq 3$.

And the $\alpha$-cut of $\bar{x}_{3}$ is $\bar{x}_{3}[\alpha]=\left[x_{3 L}(\alpha), x_{3 U}(\alpha)\right]$. Then the $\alpha$-cut of $\bar{x}_{3}$ is

$$
\begin{gathered}
x_{3 L}(\alpha)=\min \left\{\frac{a_{11}\left(a_{22} b_{3}-a_{32} b_{2}\right)-a_{12}\left(a_{21} b_{3}-a_{31} b_{2}\right)+b_{1}\left(a_{21} a_{32}-a_{31} a_{22}\right)}{a_{11}\left(a_{22} a_{33}-a_{32} a_{23}\right)-a_{12}\left(a_{21} a_{33}-a_{31} a_{23}\right)+a_{13}\left(a_{21} a_{32}-a_{31} a_{22}\right)} ;\right. \\
\left.a_{i j} \in \bar{a}_{i j}[\alpha], b_{j} \in \bar{b}_{j}[\alpha]\right\} \\
x_{3 U}(\alpha)=\max \left\{\frac{a_{11}\left(a_{22} b_{3}-a_{32} b_{2}\right)-a_{12}\left(a_{21} b_{3}-a_{31} b_{2}\right)+b_{1}\left(a_{21} a_{32}-a_{31} a_{22}\right)}{a_{11}\left(a_{22} a_{33}-a_{32} a_{23}\right)-a_{12}\left(a_{21} a_{33}-a_{31} a_{23}\right)+a_{13}\left(a_{21} a_{32}-a_{31} a_{22}\right)} ;\right. \\
\left.a_{i j} \in \bar{a}_{i j}[\alpha], b_{j} \in \bar{b}_{j}[\alpha]\right\}
\end{gathered}
$$

for $\alpha \in[0,1]$ and $1 \leq i, j \leq 3$.

After solving for the $x_{i L}(\alpha)$ and $x_{i U}(\alpha), i=1,2,3, \alpha \in[0,1]$ we check to see if the intervals $\bar{x}_{i}=\left[x_{i L}(\alpha), x_{i U}(\alpha)\right], i=1,2,3, \alpha \in[0,1]$ define continuous fuzzy numbers for $i=1,2$. What is needed is:

1) $\frac{\partial}{\partial \alpha}\left(x_{i L}(\alpha)\right)>0$,

2) $\frac{\partial}{\partial \alpha}\left(x_{i U}(\alpha)\right)<0$, and

3) $x_{i L}(1) \leq x_{i U}(1)$ for $i=1,2,3$ (equality for triangular shaped fuzzy numbers).

Now by setting $\quad \bar{X}_{e}=\left[\begin{array}{c}\bar{X}_{1} \\ \bar{X}_{2} \\ \bar{X}_{3}\end{array}\right]$ we can check whether $\bar{A} \cdot \bar{X}_{e}=\bar{B}$ is true or false.

If we set $\alpha=1$, we get the crisp solution $x_{1}$ from Equation (21) and Equation (22); crisp solution $x_{2}$ from Equation (23) and Equation (24) and crisp solution $x_{3}$ from Equation (25) and Equation (26) by assuming all the $\bar{a}_{i j}$ and $\bar{b}_{j}$ are triangular shaped fuzzy numbers and $x_{i L}(1)=x_{i U}(1)=x_{i}, i=1,2,3$.

\section{3. $\alpha$-Cuts and Interval Arithmetic}

We denote the $\alpha$-cut and interval arithmetic solution of a system of fuzzy linear equations by $\bar{X}_{I}$ and it always exists but may, or may not satisfy the original system of fuzzy linear equations. That is, $\bar{A} \cdot \bar{X}_{I}=\bar{B}$ may, or may not be true.

Let the components of $\bar{X}_{I}$ are $\bar{X}_{1}, \bar{X}_{2}$ and $\bar{X}_{3}$. In this method, we need to fuzzify the crisp solutions

$$
\begin{aligned}
& x_{1}=\frac{b_{1}\left(a_{22} a_{33}-a_{23} a_{32}\right)-a_{12}\left(a_{33} b_{2}-a_{23} b_{3}\right)+a_{13}\left(a_{32} b_{2}-a_{22} b_{3}\right)}{a_{11}\left(a_{22} a_{33}-a_{32} a_{23}\right)-a_{12}\left(a_{21} a_{33}-a_{31} a_{23}\right)+a_{13}\left(a_{21} a_{32}-a_{31} a_{22}\right)} \\
& x_{2}=\frac{a_{11}\left(a_{33} b_{2}-a_{23} b_{3}\right)-b_{1}\left(a_{21} a_{33}-a_{31} a_{23}\right)+a_{13}\left(a_{21} b_{3}-a_{31} b_{2}\right)}{a_{11}\left(a_{22} a_{33}-a_{32} a_{23}\right)-a_{12}\left(a_{21} a_{33}-a_{31} a_{23}\right)+a_{13}\left(a_{21} a_{32}-a_{31} a_{22}\right)} \\
& x_{3}=\frac{a_{11}\left(a_{22} b_{3}-a_{32} b_{2}\right)-a_{12}\left(a_{21} b_{3}-a_{31} b_{2}\right)+b_{1}\left(a_{21} a_{32}-a_{31} a_{22}\right)}{a_{11}\left(a_{22} a_{33}-a_{32} a_{23}\right)-a_{12}\left(a_{21} a_{33}-a_{31} a_{23}\right)+a_{13}\left(a_{21} a_{32}-a_{31} a_{22}\right)}
\end{aligned}
$$

using $\alpha$-cut and interval arithmetic. 
We substitute the $\alpha$-cuts of $\bar{a}_{11}, \bar{a}_{12}, \bar{a}_{13}, \bar{a}_{21}, \bar{a}_{22}, \bar{a}_{23}, \bar{a}_{31}, \bar{a}_{32}, \bar{a}_{33}, \bar{b}_{1}, \bar{b}_{2}, \bar{b}_{3}$ for $a_{11}, a_{12}, a_{13}, a_{21}, a_{22}, a_{23}, a_{31}, a_{32}, a_{33}, b_{1}, b_{2}, b_{3}$ in Equations (27)-(29) and the simplifying using interval arithmetic we obtain the $\alpha$-cuts of $\bar{x}_{1}, \bar{x}_{2}$ and $\bar{x}_{3}$.

It should be noted that

$$
\Delta=a_{11}\left(a_{22} a_{33}-a_{32} a_{23}\right)-a_{12}\left(a_{21} a_{33}-a_{31} a_{23}\right)+a_{13}\left(a_{21} a_{32}-a_{31} a_{22}\right) \neq 0,
$$

that is, the determinant of the coefficients matrix must be nonsingular and invertible.

Let $\alpha$-cut of $\bar{x}_{1}$ is $\bar{x}_{1}[\alpha]=\left[x_{1 L}(\alpha), x_{1 U}(\alpha)\right]$.

To find the $\alpha$-cut of $\bar{x}_{1}$ we substitute

$$
\bar{a}_{11}, \bar{a}_{12}, \bar{a}_{13}, \bar{a}_{21}, \bar{a}_{22}, \bar{a}_{23}, \bar{a}_{31}, \bar{a}_{32}, \bar{a}_{33}, \bar{b}_{1}, \bar{b}_{2}, \bar{b}_{3}
$$

for $a_{11}, a_{12}, a_{13}, a_{21}, a_{22}, a_{23}, a_{31}, a_{32}, a_{33}, b_{1}, b_{2}, b_{3}$ in Equation (27). Then we get,

$$
\bar{x}_{1}[\alpha]=\frac{\bar{b}_{1}[\alpha]\left(\bar{a}_{22}[\alpha] \bar{a}_{33}[\alpha]-\bar{a}_{23}[\alpha] \bar{a}_{32}[\alpha]\right)-\bar{a}_{12}[\alpha]\left(\bar{a}_{33}[\alpha] \bar{b}_{2}[\alpha]-\bar{a}_{23}[\alpha] \bar{b}_{3}[\alpha]\right)+\bar{a}_{13}[\alpha]\left(\bar{a}_{32}[\alpha] \bar{b}_{2}[\alpha]-\bar{a}_{22}[\alpha] \bar{b}_{3}[\alpha]\right)}{\bar{a}_{11}[\alpha]\left(\bar{a}_{22}[\alpha] \bar{a}_{33}[\alpha]-\bar{a}_{32}[\alpha] \bar{a}_{23}[\alpha]\right)-\bar{a}_{12}[\alpha]\left(\bar{a}_{21}[\alpha] \bar{a}_{33}[\alpha]-\bar{a}_{31}[\alpha] \bar{a}_{23}[\alpha]\right)+\bar{a}_{13}[\alpha]\left(\bar{a}_{21}[\alpha] \bar{a}_{32}[\alpha]-\bar{a}_{31}[\alpha] \bar{a}_{22}[\alpha]\right)}
$$

Let us assume that all $\bar{a}_{i j}>0$ and all the $\bar{b}_{j}>0$. Then by simplifying and using the interval arithmetic we get,

$$
x_{1 L}(\alpha)=\frac{\left(b_{1 L} a_{22 L} a_{33 L}-b_{1 U} a_{23 U} a_{32 U}\right)-\left(a_{12 L} a_{33 L} b_{2 L}-a_{12 U} a_{23 U} b_{3 U}\right)+\left(a_{13 L} a_{32 L} b_{2 L}-a_{13 U} a_{22 U} b_{3 U}\right)}{\left(a_{11 U} a_{22 U} a_{33 U}-a_{11 L} a_{32 L} a_{23 L}\right)-\left(a_{12 U} a_{21 U} a_{33 U}-a_{12 L} a_{31 L} a_{23 L}\right)+\left(a_{13 U} a_{21 U} a_{32 U}-a_{13 L} a_{31 L} a_{22 L}\right)}
$$

And

$$
x_{1 U}(\alpha)=\frac{\left(b_{1 U} a_{22 U} a_{33 U}-b_{1 L} a_{23 L} a_{32 L}\right)-\left(a_{12 U} a_{33 U} b_{2 U}-a_{12 L} a_{23 L} b_{3 L}\right)+\left(a_{13 U} a_{32 U} b_{2 U}-a_{13 L} a_{22 L} b_{3 L}\right)}{\left(a_{11 L} a_{32 L} a_{23 L}-a_{11 U} a_{22 U} a_{33 U}\right)-\left(a_{12 L} a_{31 L} a_{23 L}-a_{12 U} a_{21 U} a_{33 U}\right)+\left(a_{13 L} a_{31 L} a_{22 L}-a_{13 U} a_{21 U} a_{32 U}\right)}
$$

Again let $\alpha$-cut of $\bar{x}_{2}$ is $\bar{x}_{2}[\alpha]=\left[x_{2 L}(\alpha), x_{2 U}(\alpha)\right]$. To find the $\alpha$-cut of $\bar{x}_{2}$ we substtute $\bar{a}_{11}, \bar{a}_{12}, \bar{a}_{13}, \bar{a}_{21}, \bar{a}_{22}, \bar{a}_{23}, \bar{a}_{31}, \bar{a}_{32}, \bar{a}_{33}, \bar{b}_{1}, \bar{b}_{2}, \bar{b}_{3}$ for

$$
a_{11}, a_{12}, a_{13}, a_{21}, a_{22}, a_{23}, a_{31}, a_{32}, a_{33}, b_{1}, b_{2}, b_{3}
$$

in Equations (28). Then we get,

$$
\bar{x}_{2}[\alpha]=\frac{\bar{a}_{11}[\alpha]\left(\bar{a}_{33}[\alpha] \bar{b}_{2}[\alpha]-\bar{a}_{23}[\alpha] \bar{b}_{3}[\alpha]\right)-\bar{b}_{1}[\alpha]\left(\bar{a}_{21}[\alpha] \bar{a}_{33}[\alpha]-\bar{a}_{31}[\alpha] \bar{a}_{23}[\alpha]\right)+\bar{a}_{13}[\alpha]\left(\bar{a}_{21}[\alpha] \bar{b}_{3}[\alpha]-\bar{a}_{31}[\alpha] \bar{b}_{2}[\alpha]\right)}{\bar{a}_{11}[\alpha]\left(\bar{a}_{22}[\alpha] \bar{a}_{33}[\alpha]-\bar{a}_{32}[\alpha] \bar{a}_{23}[\alpha]\right)-\bar{a}_{12}[\alpha]\left(\bar{a}_{21}[\alpha] \bar{a}_{33}[\alpha]-\bar{a}_{31}[\alpha] \bar{a}_{23}[\alpha]\right)+\bar{a}_{13}[\alpha]\left(\bar{a}_{21}[\alpha] \bar{a}_{32}[\alpha]-\bar{a}_{31}[\alpha] \bar{a}_{22}[\alpha]\right)}
$$

Let us assume that all $\bar{a}_{i j}>0$ and all the $\bar{b}_{j}>0$. Then by simplifying and using the interval arithmetic we get,

$$
x_{2 L}(\alpha)=\frac{\left(a_{11 L} a_{33 L} b_{2 L}-a_{11 U} a_{23 U} b_{3 U}\right)-\left(a_{21 L} a_{33 L} b_{1 L}-a_{31 U} a_{23 U} b_{1 U}\right)+\left(a_{13 L} a_{21 L} b_{3 L}-a_{13 U} a_{31 U} b_{2 U}\right)}{\left(a_{11 U} a_{22 U} a_{33 U}-a_{11 L} a_{32 L} a_{23 L}\right)-\left(a_{12 U} a_{21 U} a_{33 U}-a_{12 L} a_{31 L} a_{23 L}\right)+\left(a_{13 U} a_{21 U} a_{32 U}-a_{13 L} a_{31 L} a_{22 L}\right)}
$$

And

$$
x_{2 U}(\alpha)=\frac{\left(a_{11 U} a_{23 U} b_{3 U}-a_{11} a_{33 L} b_{2 L}\right)-\left(a_{31 U} a_{23 U} b_{1 U}-a_{21 L} a_{33 L} b_{1 L}\right)+\left(a_{13 U} a_{31 U} b_{2 U}-a_{13 L} a_{21 L} b_{3 L}\right)}{\left(a_{11 L} a_{32 L} a_{23 L}-a_{11 U} a_{22 U} a_{33 U}\right)-\left(a_{12 L} a_{31 L} a_{23 L}-a_{12 U} a_{21 U} a_{33 U}\right)+\left(a_{13 L} a_{31 L} a_{22 L}-a_{13 U} a_{21 U} a_{32 U}\right)}
$$

And finally, let $\alpha$-cut of $\bar{x}_{3}$ is $\bar{x}_{3}[\alpha]=\left[x_{3 L}(\alpha), x_{3 U}(\alpha)\right]$. To find the $\alpha$-cut of $\bar{x}_{3}$ we substitute $\bar{a}_{11}, \bar{a}_{12}, \bar{a}_{13}, \bar{a}_{21}, \bar{a}_{22}, \bar{a}_{23}, \bar{a}_{31}, \bar{a}_{32}, \bar{a}_{33}, \bar{b}_{1}, \bar{b}_{2}, \bar{b}_{3}$ for

$$
a_{11}, a_{12}, a_{13}, a_{21}, a_{22}, a_{23}, a_{31}, a_{32}, a_{33}, b_{1}, b_{2}, b_{3}
$$

in Equations (29). Then we get, 


$$
\bar{x}_{3}[\alpha]=\frac{\bar{a}_{11}[\alpha]\left(\bar{a}_{22}[\alpha] \bar{b}_{3}[\alpha]-\bar{a}_{32}[\alpha] \bar{b}_{2}[\alpha]\right)-\bar{a}_{12}[\alpha]\left(\bar{a}_{21}[\alpha] \bar{b}_{3}[\alpha]-\bar{a}_{31}[\alpha] \bar{b}_{2}[\alpha]\right)+\bar{b}_{1}[\alpha]\left(\bar{a}_{21}[\alpha] \bar{a}_{32}[\alpha]-\bar{a}_{31}[\alpha] \bar{a}_{22}[\alpha]\right)}{\bar{a}_{11}[\alpha]\left(\bar{a}_{22}[\alpha] \bar{a}_{33}[\alpha]-\bar{a}_{32}[\alpha] \bar{a}_{23}[\alpha]\right)-\bar{a}_{12}[\alpha]\left(\bar{a}_{21}[\alpha] \bar{a}_{33}[\alpha]-\bar{a}_{31}[\alpha] \bar{a}_{23}[\alpha]\right)+\bar{a}_{13}[\alpha]\left(\bar{a}_{21}[\alpha] \bar{a}_{32}[\alpha]-\bar{a}_{31}[\alpha] \bar{a}_{22}[\alpha]\right)}
$$

Let us assume that all $\bar{a}_{i j}>0$ and all the $\bar{b}_{j}>0$. Then by simplifying and using the interval arithmetic we get,

$$
\begin{aligned}
& x_{3 L}(\alpha)=\frac{\left(a_{11 L} a_{22 L} b_{3 L}-a_{11 U} a_{32 U} b_{2 U}\right)-\left(a_{12 L} a_{21 L} b_{3 L}-a_{12 U} a_{31 U} b_{2 U}\right)+\left(a_{21 L} a_{32 L} b_{1 L}-a_{31 U} a_{22 U} b_{1 U}\right)}{\left(a_{11 U} a_{22 U} a_{33 U}-a_{11 L} a_{32 L} a_{23 L}\right)-\left(a_{12 U} a_{21 U} a_{33 U}-a_{12 L} a_{31 L} a_{23 L}\right)+\left(a_{13 U} a_{21 U} a_{32 U}-a_{13 L} a_{31 L} a_{22 L}\right)} \\
& \text { And } \\
& x_{3 U}(\alpha)=\frac{\left(a_{11 U} a_{32 U} b_{2 U}-a_{11 L} a_{22 L} b_{3 L}\right)-\left(a_{12 U} a_{31 U} b_{2 U}-a_{12 L} a_{21 L} b_{3 L}\right)+\left(a_{31 U} a_{22 U} b_{1 U}-a_{21 L} a_{32 L} b_{1 L}\right)}{\left(a_{11 L} a_{32 L} a_{23 L}-a_{11 U} a_{22 U} a_{33 U}\right)-\left(a_{12 L} a_{31 L} a_{23 L}-a_{12 U} a_{21 U} a_{33 U}\right)+\left(a_{13 L} a_{31 L} a_{22 L}-a_{13 U} a_{21 U} a_{32 U}\right)}
\end{aligned}
$$

After solving for the $x_{i L}(\alpha)$ and $x_{i U}(\alpha), i=1,2,3, \alpha \in[0,1]$ we check to see if the intervals $\bar{x}_{i}=\left[x_{i L}(\alpha), x_{i U}(\alpha)\right], \quad i=1,2,3, \alpha \in[0,1]$ define continuous fuzzy numbers for $i=1,2$. What is needed is:

1) $\frac{\partial}{\partial \alpha}\left(x_{i L}(\alpha)\right)>0$,

2) $\frac{\partial}{\partial \alpha}\left(x_{i U}(\alpha)\right)<0$, and

3) $x_{i L}(1) \leq x_{i U}(1)$ for $i=1,2,3$ (equality for triangular shaped fuzzy numbers).

Now by setting $\quad \bar{X}_{I}=\left[\begin{array}{l}\bar{x}_{1} \\ \bar{x}_{2} \\ \bar{x}_{3}\end{array}\right]$ we can check whether $\bar{A} \cdot \bar{X}_{I}=\bar{B}$ is true or false.

If we set $\alpha=1$, we get the crisp solution $x_{1}$ from Equation (30) and Equation (31); crisp solution $x_{2}$ from Equation (32) and Equation (33) and crisp solution $x_{3}$ from Equation (34) and Equation (35) by assuming all the $\bar{a}_{i j}$ and $\bar{b}_{j}$ are triangular shaped fuzzy numbers and $x_{i L}(1)=x_{i U}(1)=x_{i}, i=1,2,3$.

\section{Applications}

\subsection{Classical Method}

Consider the system of fuzzy linear equation in matrix form

$$
\left[\begin{array}{ccc}
(1 / 2 / 3) & 0 & 0 \\
0 & (3 / 4 / 5) & 0 \\
0 & 0 & (6 / 8 / 12)
\end{array}\right]\left[\begin{array}{c}
\bar{x}_{1} \\
\bar{x}_{2} \\
\bar{x}_{3}
\end{array}\right]=\left[\begin{array}{c}
(-1 / 1 / 2) \\
(1 / 2 / 3) \\
(2 / 5 / 8)
\end{array}\right]
$$

We solve this fuzzy matrix equation using the classical method.

The above system can be written as

$$
\left.\begin{array}{l}
(1 / 2 / 3) \bar{x}_{1}=(-1 / 1 / 2) \\
(3 / 4 / 5) \bar{x}_{2}=(1 / 2 / 3) \\
(6 / 8 / 12) \bar{x}_{3}=(2 / 5 / 8)
\end{array}\right\}
$$

Here $\bar{a}_{11}=(1 / 2 / 3), \bar{a}_{22}=(3 / 4 / 5)$, and $\bar{a}_{33}=(6 / 8 / 12)$.

Also, we have, $\bar{b}_{1}=(-1 / 1 / 2), \bar{b}_{2}=(1 / 2 / 3)$ and $\bar{b}_{3}=(2 / 5 / 8)$.

Then the $\alpha$-cuts are: 


$$
\begin{gathered}
\bar{a}_{11}[\alpha]=\left[a_{11 L}(\alpha), a_{11 U}(\alpha)\right]=[1+\alpha, 3-\alpha], \\
\bar{a}_{22}[\alpha]=\left[a_{22 L}(\alpha), a_{22 U}(\alpha)\right]=[3+\alpha, 5-\alpha], \\
\bar{a}_{33}[\alpha]=\left[a_{33 L}(\alpha), a_{33 U}(\alpha)\right]=[6+2 \alpha, 12-4 \alpha], \\
\bar{b}_{1}[\alpha]=\left[b_{1 L}(\alpha), b_{1 U}(\alpha)\right]=[-1+2 \alpha, 2-\alpha], \\
\bar{b}_{2}[\alpha]=\left[b_{2 L}(\alpha), a_{2 U}(\alpha)\right]=[1+\alpha, 3-\alpha], \\
\bar{b}_{3}[\alpha]=\left[b_{3 L}(\alpha), a_{3 U}(\alpha)\right]=[2+3 \alpha, 8-3 \alpha], \forall \alpha \in[0,1] .
\end{gathered}
$$

Now substituting the $\alpha$-cuts of $\bar{a}_{11}, \bar{a}_{22}, \bar{a}_{33}, \bar{b}_{1}, \bar{b}_{2}$ and $\bar{b}_{3}$ for $\bar{a}_{11}, \bar{a}_{22}$, $\bar{a}_{33}, \bar{b}_{1}, \bar{b}_{2}$ and $\bar{b}_{3}$ in the system (36) and we get

$$
\left.\begin{array}{l}
{[1+\alpha, 3-\alpha]\left[x_{1 L}(\alpha), x_{1 U}(\alpha)\right]=[-1+2 \alpha, 2-\alpha]} \\
{[3+\alpha, 5-\alpha]\left[x_{2 L}(\alpha), x_{2 U}(\alpha)\right]=[1+\alpha, 3-\alpha]} \\
{[6+2 \alpha, 12-4 \alpha]\left[x_{3 L}(\alpha), x_{3 U}(\alpha)\right]=[2+3 \alpha, 8-3 \alpha]}
\end{array}\right\}
$$

If we put $\alpha=1$, we get the crisp solutions $x_{1}=1 / 2, x_{2}=1 / 2$ and $x_{3}=5 / 8$. So we assume we can get a solution with $\bar{x}_{1}>0, \bar{x}_{1}[1]=3 / 2, \bar{x}_{2}>0, \bar{x}_{2}[1]=5 / 4$ and $\bar{x}_{3}>0, \bar{x}_{3}[1]=5 / 8$.

From Equation (37) we get,

$$
\left.\begin{array}{l}
(1+\alpha) x_{1 L}(\alpha)=(-1+2 \alpha) \text { or } x_{1 L}(\alpha)=\frac{(-1+2 \alpha)}{(1+\alpha)} \\
(3+\alpha) x_{2 L}(\alpha)=(1+\alpha) \text { or } x_{2 L}(\alpha)=\frac{(1+\alpha)}{(3+\alpha)} \\
(6+2 \alpha) x_{3 L}(\alpha)=(2+3 \alpha) \text { or } x_{3 L}(\alpha)=\frac{(2+3 \alpha)}{(6+2 \alpha)} \\
(3-\alpha) x_{1 U}(\alpha)=(2-\alpha) \text { or } x_{1 U}(\alpha)=\frac{(2-\alpha)}{(3-\alpha)} \\
(5-\alpha) x_{2 L}(\alpha)=(3-\alpha) \text { or } x_{2 U}(\alpha)=\frac{(3-\alpha)}{(5-\alpha)} \\
(12-4 \alpha) x_{3 U}(\alpha)=(8-3 \alpha) \text { or } x_{3 U}(\alpha)=\frac{(8-3 \alpha)}{(12-4 \alpha)}
\end{array}\right\}
$$

We find that,

$$
\begin{gathered}
\frac{\partial}{\partial \alpha}\left(x_{1 L}(\alpha)\right)=\frac{3}{(1+\alpha)^{2}}>0 ; \frac{\partial}{\partial \alpha}\left(x_{2 L}(\alpha)\right)=\frac{2}{(1+\alpha)^{2}}>0 ; \\
\frac{\partial}{\partial \alpha}\left(x_{3 L}(\alpha)\right)=\frac{14}{(6+2 \alpha)^{2}}>0 ; \frac{\partial}{\partial \alpha}\left(x_{1 U}(\alpha)\right)=-\frac{1}{(3-\alpha)^{2}}<0 ; \\
\frac{\partial}{\partial \alpha}\left(x_{2 U}(\alpha)\right)=-\frac{2}{(5-\alpha)^{2}}<0 ; \frac{\partial}{\partial \alpha}\left(x_{3 U}(\alpha)\right)=-\frac{4}{(12-4 \alpha)^{2}}<0 .
\end{gathered}
$$

That is $x_{1 L}(\alpha), x_{2 L}(\alpha)$ and $x_{3 L}(\alpha)$ are increasing functions of $\alpha \in[0,1]$ and $x_{1 U}(\alpha), x_{2 U}(\alpha)$ and $x_{3 U}(\alpha)$ are decreasing functions of $\alpha \in[0,1]$. Al- 
so $x_{1 L}(1)=x_{1 U}(1), x_{2 L}(1)=x_{2 U}(1)$ and $x_{3 L}(1)=x_{3 U}(1)$.

Hence,

$$
\bar{x}_{1}[\alpha]=\left[\frac{(-1+2 \alpha)}{(1+\alpha)}, \frac{(2-\alpha)}{(3-\alpha)}\right], \quad \bar{x}_{2}[\alpha]=\left[\frac{(1+\alpha)}{(3+\alpha)}, \frac{(3-\alpha)}{(5-\alpha)}\right]
$$

and $\bar{x}_{3}[\alpha]=\left[\frac{(2+3 \alpha)}{(6+2 \alpha)}, \frac{(8-3 \alpha)}{(12-4 \alpha)}\right]$ defines the $\alpha$-cuts of three fuzzy numbers respectively.

Now the support of $\bar{x}_{1}$ is $\bar{x}_{1}[0]=\left[\frac{(-1+2 \times 0)}{(1+0)}, \frac{(2-0)}{(3-0)}\right]=\left[-1, \frac{2}{3}\right]$ and modal of $\bar{x}_{1}$ is $\bar{x}_{1}[1]=\left[\frac{(-1+2 \times 1)}{(1+1)}, \frac{(2-1)}{(3-1)}\right]=\left[\frac{1}{2}, \frac{1}{2}\right]=\frac{1}{2}$;

The support of $\bar{x}_{2}$ is $\bar{x}_{2}[0]=\left[\frac{(1+0)}{(3+0)}, \frac{(3-0)}{(5-0)}\right]=\left[\frac{1}{3}, \frac{3}{5}\right]$ and modal of $\bar{x}_{2}$ is $\bar{x}_{2}[1]=\left[\frac{(1+1)}{(3+1)}, \frac{(3-1)}{(5-1)}\right]=\left[\frac{1}{2}, \frac{1}{2}\right]=\frac{1}{2}$; and

The support of $\bar{x}_{3}$ is $\bar{x}_{3}[0]=\left[\frac{(2+3 \times 0)}{(6+2 \times 0)}, \frac{(8-3 \times 0)}{(12-4 \times 0)}\right]=\left[\frac{1}{3}, \frac{2}{3}\right]$ and modal of $\bar{x}_{3}$ is $\bar{x}_{3}[1]=\left[\frac{(2+3 \times 1)}{(6+2 \times 1)}, \frac{(8-3 \times 1)}{(12-4 \times 1)}\right]=\left[\frac{5}{8}, \frac{5}{8}\right]=\frac{5}{8}$.

Therefore we can say that, the classical solution $\bar{X}_{c}$ exists and its components are continuous triangular shaped fuzzy numbers

$$
\bar{x}_{1} \approx\left(-1 / \frac{1}{2} / \frac{2}{3}\right), \bar{x}_{2} \approx\left(\frac{1}{3} / \frac{1}{2} / \frac{3}{5}\right) \text { and } \bar{x}_{3} \approx\left(\frac{1}{3} / \frac{5}{8} / \frac{2}{3}\right) .
$$

The membership function of the triangularly shaped number $\bar{x}_{1} \approx\left(-1 / \frac{1}{2} / \frac{2}{3}\right)$ is

$$
x=\frac{(-1+2 \alpha)}{(1+\alpha)} \Rightarrow \mu_{1 L}(x)=\alpha=\frac{(1+x)}{(2-x)}, \text { for }-1 \leq x \leq \frac{1}{2}
$$

and,

$$
x=\frac{(2-\alpha)}{(3-\alpha)}=\mu_{1 U}(x)=\alpha=\frac{(2-3 x)}{(1-x)}, \text { for } \frac{1}{2} \leq x \leq \frac{2}{3} .
$$

Thus the membership function of $\bar{x}_{1} \approx\left(-1 / \frac{1}{2} / \frac{2}{3}\right)$ is

$$
\mu_{\bar{x}_{1}}(x)= \begin{cases}\frac{(1+x)}{(2-x)}, & \text { for }-1 \leq x \leq \frac{1}{2} \\ \frac{(2-3 x)}{(1-x)}, & \text { for } \frac{1}{2} \leq x \leq \frac{2}{3} \\ 0, & \text { otherwise }\end{cases}
$$


and its graph is shown in Figure 1.

The membership function of the triangularly shaped number $\bar{x}_{2} \approx\left(\frac{1}{3} / \frac{1}{2} / \frac{3}{5}\right)$ is

$$
x=\frac{(1+\alpha)}{(3+\alpha)} \Rightarrow \mu_{2 L}(x)=\alpha=\frac{(1-3 x)}{(x-1)}, \text { for } \frac{1}{3} \leq x \leq \frac{1}{2},
$$

and

$$
x=\frac{(3-\alpha)}{(5-\alpha)}=\mu_{2 U}(x)=\alpha=\frac{(3-5 x)}{(1-x)}, \text { for } \frac{1}{2} \leq x \leq \frac{3}{5} .
$$

Thus the membership function of $\bar{x}_{2} \approx\left(\frac{1}{3} / \frac{1}{2} / \frac{3}{5}\right)$ is

$$
\mu_{\bar{x}_{2}}(x)= \begin{cases}\frac{(1-3 x)}{(x-1)}, & \text { for } \frac{1}{3} \leq x \leq \frac{1}{2} \\ \frac{(3-5 x)}{(1-x)}, & \text { for } \frac{1}{2} \leq x \leq \frac{3}{5} \\ 0, & \text { otherwise }\end{cases}
$$

and its graph is shown in Figure 2.

And the membership function of the triangular shaped number $\bar{x}_{3} \approx\left(\frac{1}{3} / \frac{5}{8} / \frac{2}{3}\right)$ is

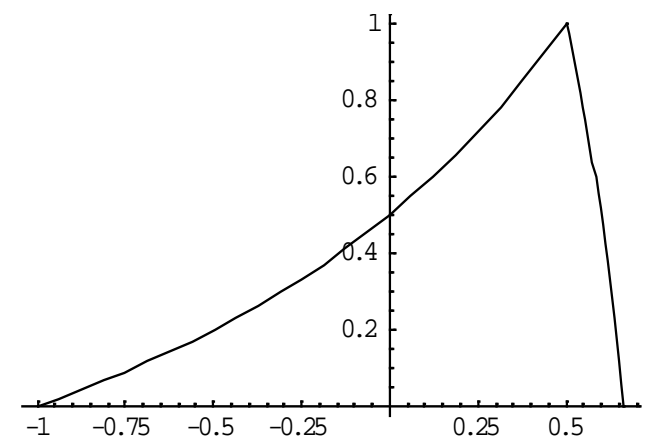

Figure 1. Graph of the membership function of $\bar{x}_{1} \approx\left(-1 / \frac{1}{2} / \frac{2}{3}\right)$.

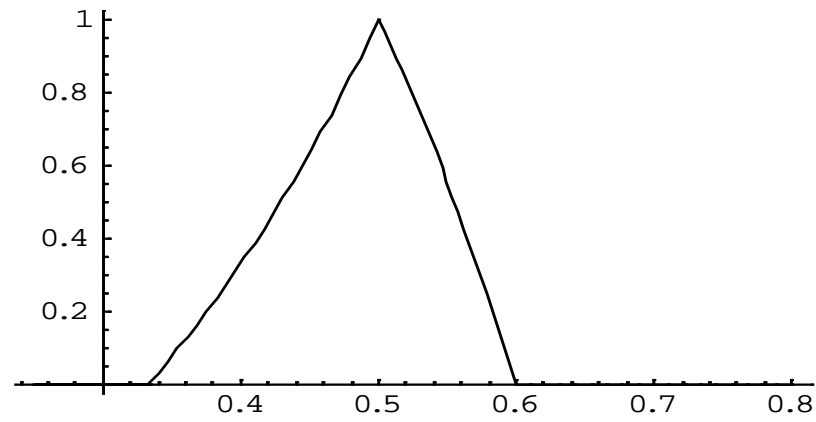

Figure 2. Graph of the membership function of $\bar{x}_{2} \approx\left(\frac{1}{3} / \frac{1}{2} / \frac{3}{5}\right)$. 


$$
x=\frac{(2+3 \alpha)}{(6+2 \alpha)} \Rightarrow \mu_{3 L}(x)=\alpha=\frac{(2-6 x)}{(2 x-3)}, \text { for } \frac{1}{3} \leq x \leq \frac{5}{8},
$$

and

$$
x=\frac{(8-3 \alpha)}{(12-4 \alpha)}=\mu_{3 U}(x)=\alpha=\frac{(8-12 x)}{(3-4 x)} \text {, for } \frac{5}{8} \leq x \leq \frac{2}{3} .
$$

Thus the membership function of $\bar{x}_{3} \approx\left(\frac{1}{3} / \frac{5}{8} / \frac{2}{3}\right)$ is

$$
\mu_{\bar{x}_{3}}(x)= \begin{cases}\frac{(2-6 x)}{(2 x-3)}, & \text { for } \frac{1}{3} \leq x \leq \frac{5}{8} \\ \frac{(8-12 x)}{(3-4 x)}, & \text { for } \frac{5}{8} \leq x \leq \frac{2}{3} \\ 0, & \text { otherwise }\end{cases}
$$

and its graph is shown in Figure 3.

Finally the graph of the classical solution

$$
\bar{X}_{c}=\left[\begin{array}{l}
\bar{X}_{1} \approx\left(-1 / \frac{1}{2} / \frac{2}{3}\right) \\
\bar{x}_{2} \approx\left(\frac{1}{3} / \frac{1}{2} / \frac{3}{5}\right) \\
\bar{x}_{3} \approx\left(\frac{1}{3} / \frac{5}{8} / \frac{2}{3}\right)
\end{array}\right]
$$

is shown in Figure 4.

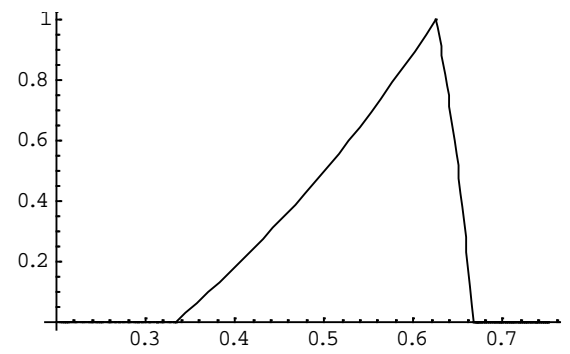

Figure 3. Graph of the membership function of $\bar{x}_{3} \approx\left(\frac{1}{3} / \frac{5}{8} / \frac{2}{3}\right)$.

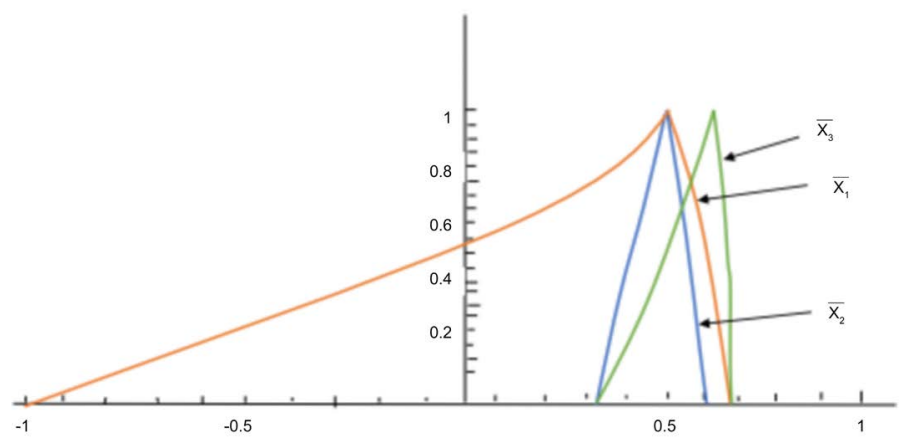

Figure 4. Graph of the classical solution $\bar{X}_{c}$. 


\subsection{Extension Principle Method}

Consider the system of fuzzy linear equation in matrix form

$$
\left[\begin{array}{ccc}
(1 / 2 / 3) & 0 & 0 \\
0 & (3 / 4 / 5) & 0 \\
0 & 0 & (6 / 8 / 12)
\end{array}\right]\left[\begin{array}{l}
\bar{x}_{1} \\
\bar{x}_{2} \\
\bar{x}_{3}
\end{array}\right]=\left[\begin{array}{c}
(-1 / 1 / 2) \\
(1 / 2 / 3) \\
(2 / 5 / 8)
\end{array}\right]
$$

We solve this fuzzy matrix equation using the extension principle method.

Here, $\quad \bar{a}_{11}=(1 / 2 / 3), \quad \bar{a}_{12}=0, \quad \bar{a}_{13}=0, \bar{a}_{21}=0, \bar{a}_{22}=(3 / 4 / 5), \quad \bar{a}_{23}=0$, $\bar{a}_{31}=0, \quad \bar{a}_{32}=0$ and $\bar{a}_{33}=(6 / 8 / 12)$. Also, we have, $\bar{b}_{1}=(-1 / 1 / 2)$, $\bar{b}_{2}=(1 / 2 / 3)$ and $\bar{b}_{3}=(2 / 5 / 8)$.

Then the $\alpha$-cuts are:

$$
\begin{gathered}
\bar{a}_{11}[\alpha]=\left[a_{11 L}(\alpha), a_{11 U}(\alpha)\right]=[1+\alpha, 3-\alpha], \\
\bar{a}_{22}[\alpha]=\left[a_{22 L}(\alpha), a_{22 U}(\alpha)\right]=[3+\alpha, 5-\alpha], \\
\bar{a}_{33}[\alpha]=\left[a_{33 L}(\alpha), a_{33 U}(\alpha)\right]=[6+2 \alpha, 12-4 \alpha], \\
\bar{b}_{1}[\alpha]=\left[b_{1 L}(\alpha), b_{1 U}(\alpha)\right]=[-1+2 \alpha, 2-\alpha], \\
\bar{b}_{2}[\alpha]=\left[b_{2 L}(\alpha), a_{2 U}(\alpha)\right]=[1+\alpha, 3-\alpha], \\
\bar{b}_{3}[\alpha]=\left[b_{3 L}(\alpha), a_{3 U}(\alpha)\right]=[2+3 \alpha, 8-3 \alpha], \forall \alpha \in[0,1] .
\end{gathered}
$$

Now the crisp solutions are

$$
\begin{gathered}
x_{1}=\frac{b_{1}\left(a_{22} a_{33}-a_{23} a_{32}\right)-a_{12}\left(a_{33} b_{2}-a_{23} b_{3}\right)+a_{13}\left(a_{32} b_{2}-a_{22} b_{3}\right)}{a_{11}\left(a_{22} a_{33}-a_{32} a_{23}\right)-a_{12}\left(a_{21} a_{33}-a_{31} a_{23}\right)+a_{13}\left(a_{21} a_{32}-a_{31} a_{22}\right)}=\frac{a_{22} a_{33} b_{1}}{a_{11} a_{22} a_{33}} \\
\therefore h_{1}\left(a_{11}, a_{12}, a_{13}, a_{21}, a_{22}, a_{23}, a_{31}, a_{32}, a_{33}, b_{1}, b_{2}, b_{3}\right)=\frac{b_{1}}{a_{11}} \\
x_{2}=\frac{a_{11}\left(a_{33} b_{2}-a_{23} b_{3}\right)-b_{1}\left(a_{21} a_{33}-a_{31} a_{23}\right)+a_{13}\left(a_{21} b_{3}-a_{31} b_{2}\right)}{a_{11}\left(a_{22} a_{33}-a_{32} a_{23}\right)-a_{12}\left(a_{21} a_{33}-a_{31} a_{23}\right)+a_{13}\left(a_{21} a_{32}-a_{31} a_{22}\right)}=\frac{a_{11} a_{33} b_{2}}{a_{11} a_{22} a_{33}} \\
\therefore h_{2}\left(a_{11}, a_{12}, a_{13}, a_{21}, a_{22}, a_{23}, a_{31}, a_{32}, a_{33}, b_{1}, b_{2}, b_{3}\right)=\frac{b_{2}}{a_{22}} \\
x_{3}=\frac{a_{11}\left(a_{22} b_{3}-a_{32} b_{2}\right)-a_{12}\left(a_{21} b_{3}-a_{31} b_{2}\right)+b_{1}\left(a_{21} a_{32}-a_{31} a_{22}\right)}{a_{11}\left(a_{22} a_{33}-a_{32} a_{23}\right)-a_{12}\left(a_{21} a_{33}-a_{31} a_{23}\right)+a_{13}\left(a_{21} a_{32}-a_{31} a_{22}\right)}=\frac{a_{11} a_{22} b_{3}}{a_{11} a_{22} a_{33}} \\
\therefore h_{3}\left(a_{11}, a_{12}, a_{13}, a_{21}, a_{22}, a_{23}, a_{31}, a_{32}, a_{33}, b_{1}, b_{2}, b_{3}\right)=\frac{b_{3}}{a_{33}}
\end{gathered}
$$

Since $\frac{b_{1}}{a_{11}}, \frac{b_{2}}{a_{22}}$ and $\frac{b_{3}}{a_{33}}$ are increasing functions of $b_{1}, b_{2}$ and $b_{3}$; and decreasing functions of $a_{11}, a_{22}$ and $a_{33}$, then

$$
\begin{gathered}
\min \left\{\frac{b_{1}}{a_{11}}: b_{1} \in \bar{b}_{1}[\alpha], a_{11} \in \bar{a}_{11}[\alpha]\right\}=\frac{b_{1 L}(\alpha)}{a_{11 U}(\alpha)} \\
\text { and } \max \left\{\frac{b_{1}}{a_{11}}: b_{1} \in \bar{b}_{1}[\alpha], a_{11} \in \bar{a}_{11}[\alpha]\right\}=\frac{b_{1 U}(\alpha)}{a_{11 L}(\alpha)}, \forall \alpha \in[0,1] .
\end{gathered}
$$




$$
\therefore \bar{x}_{1}[\alpha]=\left[\frac{b_{1 L}(\alpha)}{a_{11 U}(\alpha)}, \frac{b_{1 U}(\alpha)}{a_{11 L}(\alpha)}\right]=\left[\frac{-1+2 \alpha}{3-\alpha}, \frac{2-\alpha}{1+\alpha}\right]
$$

Similarly,

$$
\bar{x}_{2}[\alpha]=\left[\frac{b_{2 L}(\alpha)}{a_{22 U}(\alpha)}, \frac{b_{2 U}(\alpha)}{a_{22 L}(\alpha)}\right]=\left[\frac{1+\alpha}{5-\alpha}, \frac{3-\alpha}{3+\alpha}\right] .
$$

Also,

$$
\bar{x}_{3}[\alpha]=\left[\frac{b_{3 L}(\alpha)}{a_{33 U}(\alpha)}, \frac{b_{3 U}(\alpha)}{a_{33 L}(\alpha)}\right]=\left[\frac{2+3 \alpha}{12-4 \alpha}, \frac{8-3 \alpha}{6+2 \alpha}\right] .
$$

Here,

$$
\begin{aligned}
& x_{1 L}(\alpha)=\frac{-1+2 \alpha}{3-\alpha}, x_{2 L}(\alpha)=\frac{1+\alpha}{5-\alpha}, x_{3 L}(\alpha)=\frac{2+3 \alpha}{12-4 \alpha}, \\
& x_{1 U}(\alpha)=\frac{2-\alpha}{1+\alpha}, x_{2 U}(\alpha)=\frac{3-\alpha}{3+\alpha}, x_{3 U}(\alpha)=\frac{8-3 \alpha}{6+2 \alpha} .
\end{aligned}
$$

We find that,

$$
\begin{gathered}
\frac{\partial}{\partial \alpha}\left(x_{1 L}(\alpha)\right)=\frac{5}{(3-\alpha)^{2}}>0 ; \frac{\partial}{\partial \alpha}\left(x_{2 L}(\alpha)\right)=\frac{6}{(5-\alpha)^{2}}>0 ; \\
\frac{\partial}{\partial \alpha}\left(x_{3 L}(\alpha)\right)=\frac{44}{(12-4 \alpha)^{2}}>0 ; \frac{\partial}{\partial \alpha}\left(x_{1 U}(\alpha)\right)=-\frac{3}{(1+\alpha)^{2}}<0 ; \\
\frac{\partial}{\partial \alpha}\left(x_{2 U}(\alpha)\right)=-\frac{6}{(3+\alpha)^{2}}<0 ; \frac{\partial}{\partial \alpha}\left(x_{3 U}(\alpha)\right)=-\frac{34}{(6+2 \alpha)^{2}}<0 .
\end{gathered}
$$

That is, $x_{1 L}(\alpha), x_{2 L}(\alpha)$ and $x_{3 L}(\alpha)$ are increasing functions of $\alpha \in[0,1]$ and $x_{1 U}(\alpha), x_{2 U}(\alpha)$ and $x_{3 U}(\alpha)$ are decreasing functions of $\alpha \in[0,1]$. Also $x_{1 L}(1)=x_{1 U}(1), x_{2 L}(1)=x_{2 U}(1)$ and $x_{3 L}(1)=x_{3 U}(1)$.

Hence,

$\bar{x}_{1}[\alpha]=\left[\frac{-1+2 \alpha}{3-\alpha}, \frac{2-\alpha}{1+\alpha}\right], \quad \bar{x}_{2}[\alpha]=\left[\frac{1+\alpha}{5-\alpha}, \frac{3-\alpha}{3+\alpha}\right]$ and $\bar{x}_{3}[\alpha]=\left[\frac{2+3 \alpha}{12-4 \alpha}, \frac{8-3 \alpha}{6+2 \alpha}\right]$ define the $\alpha$-cuts of three fuzzy numbers respectively.

Now the support of $\bar{x}_{1}$ is $\bar{x}_{1}[0]=\left[\frac{-1+2 \times 0}{3-0}, \frac{2-0}{1+0}\right]=\left[-\frac{1}{3}, 2\right]$ and modal of $\bar{x}_{1}$ is $\bar{x}_{1}[1]=\left[\frac{-1+2 \times 1}{3-1}, \frac{2-1}{1+1}\right]=\left[\frac{1}{2}, \frac{1}{2}\right]=\frac{1}{2}$;

The support of $\bar{x}_{2}$ is $\bar{x}_{2}[0]=\left[\frac{1+0}{5-0}, \frac{3-0}{3+0}\right]=\left[\frac{1}{5}, 1\right]$ and modal of $\bar{x}_{2}$ is

$$
\bar{x}_{2}[1]=\left[\frac{1+1}{5-1}, \frac{3-1}{3+1}\right]=\left[\frac{1}{2}, \frac{1}{2}\right]=\frac{1}{2} ;
$$

and the support of $\bar{x}_{3}$ is $\bar{x}_{3}[0]=\left[\frac{2+3 \times 0}{12-4 \times 0}, \frac{8-3 \times 0}{6+2 \times 0}\right]=\left[\frac{1}{6}, \frac{4}{3}\right]$ and modal of $\bar{x}_{3}$ is $\bar{x}_{3}[1]=\left[\frac{2+3 \times 1}{12-4 \times 1}, \frac{8-3 \times 1}{6+2 \times 1}\right]=\left[\frac{5}{8}, \frac{5}{8}\right]=\frac{5}{8}$. 
Therefore we can say that, the extension principle solution $\bar{X}_{e}$ exists and its components are continuous triangular shaped fuzzy numbers $\bar{x}_{1} \approx\left(-\frac{1}{3} / \frac{1}{2} / 2\right)$, $\bar{x}_{2} \approx\left(\frac{1}{5} / \frac{1}{2} / 1\right)$ and $\bar{x}_{3} \approx\left(\frac{1}{6} / \frac{5}{8} / \frac{4}{3}\right)$.

The membership function of the triangularly shaped number $\bar{x}_{1} \approx\left(-\frac{1}{3} / \frac{1}{2} / 2\right)$, is

$$
x=\frac{(-1+2 \alpha)}{(3-\alpha)} \Rightarrow \mu_{1 L}(x)=\alpha=\frac{(1+3 x)}{(2+x)}, \text { for }-\frac{1}{3} \leq x \leq \frac{1}{2},
$$

and

$$
x=\frac{2-\alpha}{1+\alpha} \Rightarrow \mu_{1 U}(x)=\alpha=\frac{(2-x)}{(1+x)} \text {, for } \frac{1}{2} \leq x \leq 2 .
$$

Thus the membership function of $\bar{x}_{1} \approx\left(-\frac{1}{3} / \frac{1}{2} / 2\right)$ is

$$
\mu_{\bar{x}_{1}}(x)= \begin{cases}\frac{(1+3 x)}{(2+x)}, & \text { for }-\frac{1}{3} \leq x \leq \frac{1}{2} \\ \frac{(2-x)}{(1+x)}, & \text { for } \frac{1}{2} \leq x \leq 2 \\ 0, & \text { otherwise }\end{cases}
$$

and its graph is shown in Figure 5.

The membership function of the triangularly shaped number $\bar{x}_{2} \approx\left(\frac{1}{5} / \frac{1}{2} / 1\right)$ is

$$
x=\frac{(1+\alpha)}{(5-\alpha)} \Rightarrow \mu_{2 L}(x)=\alpha=\frac{(-1+5 x)}{(1+x)}, \text { for } \frac{1}{5} \leq x \leq \frac{1}{2},
$$

and

$$
x=\frac{(3-\alpha)}{(3+\alpha)} \Rightarrow \mu_{2 U}(x)=\alpha=\frac{(3-3 x)}{(1+x)}, \text { for } \frac{1}{2} \leq x \leq 1 .
$$

Thus the membership function of $\bar{x}_{2} \approx\left(\frac{1}{5} / \frac{1}{2} / 1\right)$ is

$$
\mu_{\bar{x}_{2}}(x)= \begin{cases}\frac{(-1+5 x)}{(1+x)}, & \text { for } \frac{1}{5} \leq x \leq \frac{1}{2} \\ \frac{(3-3 x)}{(1+x)}, & \text { for } \frac{1}{2} \leq x \leq 1 \\ 0, & \text { otherwise }\end{cases}
$$

and its graph is shown in Figure 6.

And the membership function of the triangularly shaped number $\bar{x}_{3} \approx\left(\frac{1}{6} / \frac{5}{8} / \frac{4}{3}\right)$ is 


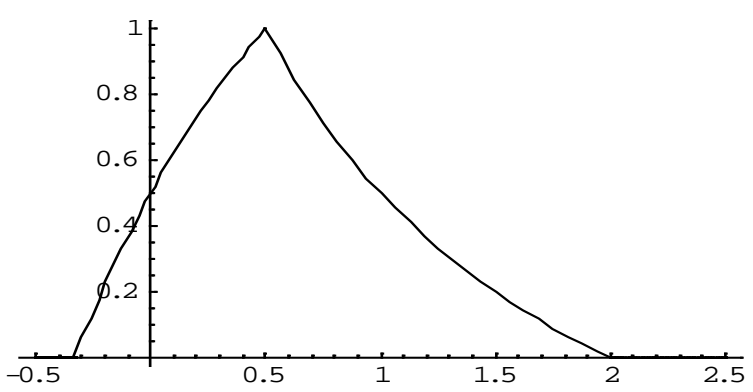

Figure 5. Graph of the membership function of $\bar{x}_{1} \approx\left(-\frac{1}{3} / \frac{1}{2} / 2\right)$.

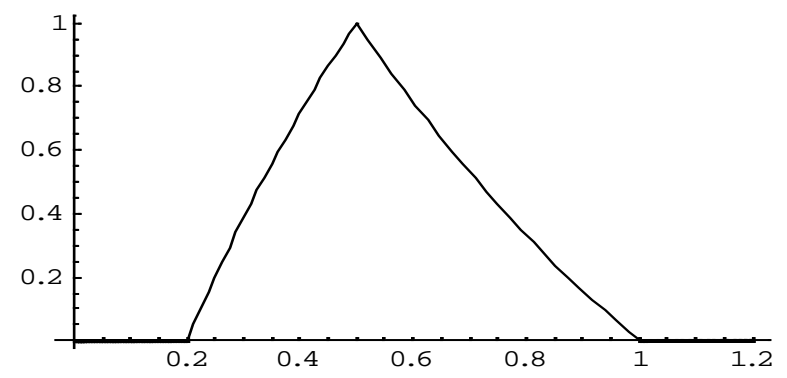

Figure 6. Graph of the membership function of $\bar{x}_{2} \approx\left(\frac{1}{5} / \frac{1}{2} / 1\right)$.

$$
x=\frac{2+3 \alpha}{12-4 \alpha} \Rightarrow \mu_{3 L}(x)=\alpha=\frac{(-2+12 x)}{(3+4 x)}, \text { for } \frac{1}{6} \leq x \leq \frac{5}{8}
$$

and

$$
x=\frac{8-3 \alpha}{6+2 \alpha} \Rightarrow \mu_{3 U}(x)=\alpha=\frac{(8-6 x)}{(3+2 x)}, \text { for } \frac{5}{8} \leq x \leq \frac{4}{3}
$$

Thus the membership function of $\bar{x}_{3} \approx\left(\frac{1}{6} / \frac{5}{8} / \frac{4}{3}\right)$ is

$$
\mu_{\bar{x}_{3}}(x)= \begin{cases}\frac{(-2+12 x)}{(3+4 x)}, & \text { for } \frac{1}{6} \leq x \leq \frac{5}{8} \\ \frac{(8-6 x)}{(3+2 x)}, & \text { for } \frac{5}{8} \leq x \leq \frac{4}{3} \\ 0, & \text { otherwise }\end{cases}
$$

and its graph is shown in Figure 7.

Finally the graph of the extension principle solution

$$
\bar{X}_{e}=\left[\begin{array}{c}
\bar{x}_{1} \approx\left(-\frac{1}{3} / \frac{1}{2} / 2\right) \\
\bar{x}_{2} \approx\left(\frac{1}{5} / \frac{1}{2} / 1\right) \\
\bar{x}_{3} \approx\left(\frac{1}{6} / \frac{5}{8} / \frac{4}{3}\right)
\end{array}\right]
$$

is shown in Figure 8. 


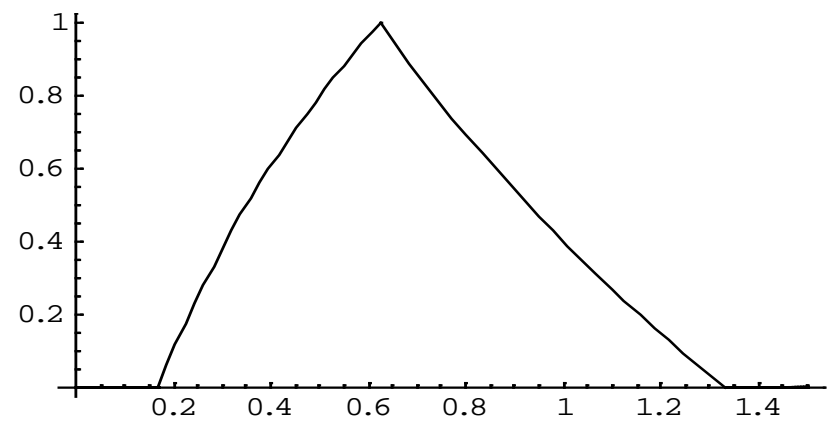

Figure 7. Graph of the membership function of $\bar{x}_{3} \approx\left(\frac{1}{6} / \frac{5}{8} / \frac{4}{3}\right)$.

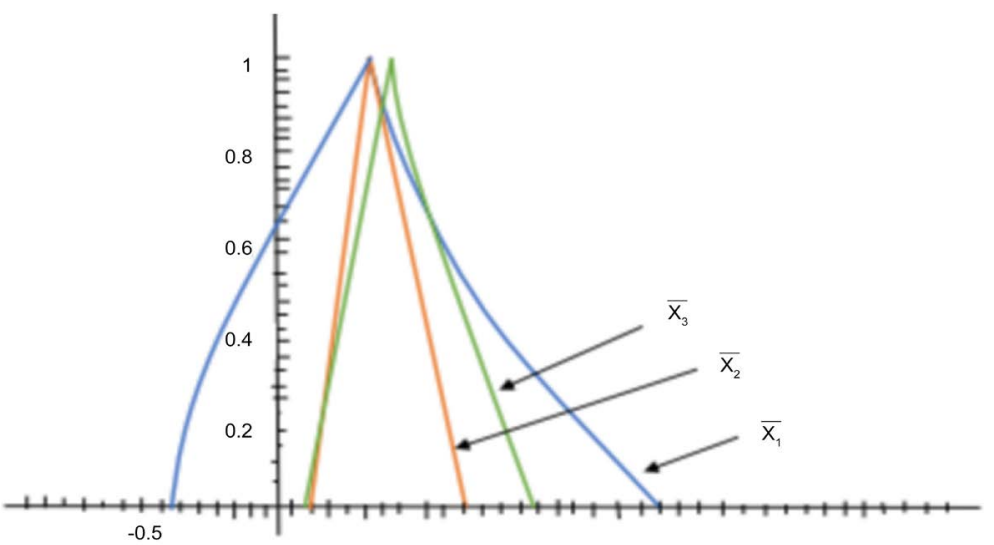

Figure 8. Graph of the extension principle solution $\bar{X}_{e}$.

\section{3. $\alpha$-Cut and Interval Arithmetic}

Consider the system of fuzzy linear equation in matrix form

$$
\left[\begin{array}{ccc}
(1 / 2 / 3) & 0 & 0 \\
0 & (3 / 4 / 5) & 0 \\
0 & 0 & (6 / 8 / 12)
\end{array}\right]\left[\begin{array}{l}
\bar{x}_{1} \\
\bar{x}_{2} \\
\bar{x}_{3}
\end{array}\right]=\left[\begin{array}{c}
(-1 / 1 / 2) \\
(1 / 2 / 3) \\
(2 / 5 / 8)
\end{array}\right]
$$

We solve this fuzzy matrix equation using $\alpha$-cut and interval arithmetic.

Here, $\quad \bar{a}_{11}=(1 / 2 / 3), \quad \bar{a}_{12}=0, \quad \bar{a}_{13}=0, \quad \bar{a}_{21}=0, \quad \bar{a}_{22}=(3 / 4 / 5), \quad \bar{a}_{23}=0$, $\bar{a}_{31}=0, \quad \bar{a}_{32}=0$ and $\bar{a}_{33}=(6 / 8 / 12)$. Also we have, $\bar{b}_{1}=(-1 / 1 / 2)$, $\bar{b}_{2}=(1 / 2 / 3)$ and $\bar{b}_{3}=(2 / 5 / 8)$.

Then the $\alpha$-cuts are:

$$
\begin{gathered}
\bar{a}_{11}[\alpha]=\left[a_{11 L}(\alpha), a_{11 U}(\alpha)\right]=[1+\alpha, 3-\alpha], \\
\bar{a}_{22}[\alpha]=\left[a_{22 L}(\alpha), a_{22 U}(\alpha)\right]=[3+\alpha, 5-\alpha], \\
\bar{a}_{33}[\alpha]=\left[a_{33 L}(\alpha), a_{33 U}(\alpha)\right]=[6+2 \alpha, 12-4 \alpha], \\
\bar{b}_{1}[\alpha]=\left[b_{1 L}(\alpha), b_{1 U}(\alpha)\right]=[-1+2 \alpha, 2-\alpha], \\
\bar{b}_{2}[\alpha]=\left[b_{2 L}(\alpha), a_{2 U}(\alpha)\right]=[1+\alpha, 3-\alpha], \\
\bar{b}_{3}[\alpha]=\left[b_{3 L}(\alpha), a_{3 U}(\alpha)\right]=[2+3 \alpha, 8-3 \alpha], \forall \alpha \in[0,1] .
\end{gathered}
$$


Now the crisp solutions are

$$
\begin{aligned}
x_{1} & =\frac{b_{1}\left(a_{22} a_{33}-a_{23} a_{32}\right)-a_{12}\left(a_{33} b_{2}-a_{23} b_{3}\right)+a_{13}\left(a_{32} b_{2}-a_{22} b_{3}\right)}{a_{11}\left(a_{22} a_{33}-a_{32} a_{23}\right)-a_{12}\left(a_{21} a_{33}-a_{31} a_{23}\right)+a_{13}\left(a_{21} a_{32}-a_{31} a_{22}\right)} \\
& =\frac{a_{22} a_{33} b_{1}}{a_{11} a_{22} a_{33}}=\frac{b_{1}}{a_{11}} \\
x_{2} & =\frac{a_{11}\left(a_{33} b_{2}-a_{23} b_{3}\right)-b_{1}\left(a_{21} a_{33}-a_{31} a_{23}\right)+a_{13}\left(a_{21} b_{3}-a_{31} b_{2}\right)}{a_{11}\left(a_{22} a_{33}-a_{32} a_{23}\right)-a_{12}\left(a_{21} a_{33}-a_{31} a_{23}\right)+a_{13}\left(a_{21} a_{32}-a_{31} a_{22}\right)} \\
& =\frac{a_{11} a_{33} b_{2}}{a_{11} a_{22} a_{33}}=\frac{b_{2}}{a_{22}} \\
x_{3} & =\frac{a_{11}\left(a_{22} b_{3}-a_{32} b_{2}\right)-a_{12}\left(a_{21} b_{3}-a_{31} b_{2}\right)+b_{1}\left(a_{21} a_{32}-a_{31} a_{22}\right)}{a_{11}\left(a_{22} a_{33}-a_{32} a_{23}\right)-a_{12}\left(a_{21} a_{33}-a_{31} a_{23}\right)+a_{13}\left(a_{21} a_{32}-a_{31} a_{22}\right)} \\
& =\frac{a_{11} a_{22} b_{3}}{a_{11} a_{22} a_{33}}=\frac{b_{3}}{a_{33}}
\end{aligned}
$$

We replace $a_{11}, a_{22}, a_{33}, b_{1}, b_{2}, b_{3}$ by $\bar{a}_{11}, \bar{a}_{22}, \bar{a}_{33}, \bar{b}_{1}, \bar{b}_{2}, \bar{b}_{3}$ respectively in Equations (48)-(50).

Then,

$\bar{x}_{1}[\alpha]=\frac{\bar{b}_{1}[\alpha]}{\bar{a}_{11}[\alpha]}=\frac{\left[b_{1 L}(\alpha), b_{1 U}(\alpha)\right]}{\left[a_{11 L}(\alpha), a_{11 U}(\alpha)\right]}=\left[\frac{b_{1 L}(\alpha)}{a_{11 U}(\alpha)}, \frac{b_{1 U}(\alpha)}{a_{11 L}(\alpha)}\right]=\left[\frac{-1+2 \alpha}{3-\alpha}, \frac{2-\alpha}{1+\alpha}\right]$

Similarly,

$\bar{x}_{2}[\alpha]=\frac{\bar{b}_{2}[\alpha]}{\bar{a}_{22}[\alpha]}=\frac{\left[b_{2 L}(\alpha), b_{2 U}(\alpha)\right]}{\left[a_{22 L}(\alpha), a_{22 U}(\alpha)\right]}=\left[\frac{b_{2 L}(\alpha)}{a_{22 U}(\alpha)}, \frac{b_{2 U}(\alpha)}{a_{22 L}(\alpha)}\right]=\left[\frac{1+\alpha}{5-\alpha}, \frac{3-\alpha}{3+\alpha}\right]$.

Also,

$\bar{x}_{3}[\alpha]=\frac{\bar{b}_{3}[\alpha]}{\bar{a}_{33}[\alpha]}=\frac{\left[b_{3 L}(\alpha), b_{3 U}(\alpha)\right]}{\left[a_{33 L}(\alpha), a_{33 U}(\alpha)\right]}=\left[\frac{b_{3 L}(\alpha)}{a_{33 U}(\alpha)}, \frac{b_{3 U}(\alpha)}{a_{33 L}(\alpha)}\right]=\left[\frac{2+3 \alpha}{12-4 \alpha}, \frac{8-3 \alpha}{6+2 \alpha}\right]$.

Here,

$$
\begin{aligned}
& x_{1 L}(\alpha)=\frac{-1+2 \alpha}{3-\alpha}, x_{2 L}(\alpha)=\frac{1+\alpha}{5-\alpha}, x_{3 L}(\alpha)=\frac{2+3 \alpha}{12-4 \alpha}, \\
& x_{1 U}(\alpha)=\frac{2-\alpha}{1+\alpha}, x_{2 U}(\alpha)=\frac{3-\alpha}{3+\alpha}, x_{3 U}(\alpha)=\frac{8-3 \alpha}{6+2 \alpha} .
\end{aligned}
$$

We find that,

$$
\begin{gathered}
\frac{\partial}{\partial \alpha}\left(x_{1 L}(\alpha)\right)=\frac{5}{(3-\alpha)^{2}}>0 ; \frac{\partial}{\partial \alpha}\left(x_{2 L}(\alpha)\right)=\frac{6}{(5-\alpha)^{2}}>0 ; \\
\frac{\partial}{\partial \alpha}\left(x_{3 L}(\alpha)\right)=\frac{44}{(12-4 \alpha)^{2}}>0 ; \frac{\partial}{\partial \alpha}\left(x_{1 U}(\alpha)\right)=-\frac{3}{(1+\alpha)^{2}}<0 ; \\
\frac{\partial}{\partial \alpha}\left(x_{2 U}(\alpha)\right)=-\frac{6}{(3+\alpha)^{2}}<0 ; \frac{\partial}{\partial \alpha}\left(x_{3 U}(\alpha)\right)=-\frac{34}{(6+2 \alpha)^{2}}<0 .
\end{gathered}
$$

That is, $x_{1 L}(\alpha), x_{2 L}(\alpha)$ and $x_{3 L}(\alpha)$ are increasing functions of $\alpha \in[0,1]$ and $x_{1 U}(\alpha), x_{2 U}(\alpha)$ and $x_{3 U}(\alpha)$ are decreasing functions of $\alpha \in[0,1]$. Also 
$x_{1 L}(1)=x_{1 U}(1), \quad x_{2 L}(1)=x_{2 U}(1)$ and $x_{3 L}(1)=x_{3 U}(1)$.

Hence,

$\bar{x}_{1}[\alpha]=\left[\frac{-1+2 \alpha}{3-\alpha}, \frac{2-\alpha}{1+\alpha}\right], \quad \bar{x}_{2}[\alpha]=\left[\frac{1+\alpha}{5-\alpha}, \frac{3-\alpha}{3+\alpha}\right]$ and $\bar{x}_{3}[\alpha]=\left[\frac{2+3 \alpha}{12-4 \alpha}, \frac{8-3 \alpha}{6+2 \alpha}\right]$

define the $\alpha$-cuts of three fuzzy numbers respectively.

Now the support of $\bar{x}_{1}$ is $\bar{x}_{1}[0]=\left[\frac{-1+2 \times 0}{3-0}, \frac{2-0}{1+0}\right]=\left[-\frac{1}{3}, 2\right]$ and modal of $\bar{x}_{1}$ is $\bar{x}_{1}[1]=\left[\frac{-1+2 \times 1}{3-1}, \frac{2-1}{1+1}\right]=\left[\frac{1}{2}, \frac{1}{2}\right]=\frac{1}{2}$;

The support of $\bar{x}_{2}$ is $\bar{x}_{2}[0]=\left[\frac{1+0}{5-0}, \frac{3-0}{3+0}\right]=\left[\frac{1}{5}, 1\right]$ and modal of $\bar{x}_{2}$ is $\bar{x}_{2}[1]=\left[\frac{1+1}{5-1}, \frac{3-1}{3+1}\right]=\left[\frac{1}{2}, \frac{1}{2}\right]=\frac{1}{2}$; and

The support of $\bar{x}_{3}$ is $\bar{x}_{3}[0]=\left[\frac{2+3.0}{12-4.0}, \frac{8-3.0}{6+2.0}\right]=\left[\frac{1}{6}, \frac{4}{3}\right]$ and modal of $\bar{x}_{3}$ is $\bar{x}_{3}[1]=\left[\frac{2+3 \times 1}{12-4 \times 1}, \frac{8-3 \times 1}{6+2 \times 1}\right]=\left[\frac{5}{8}, \frac{5}{8}\right]=\frac{5}{8}$.

Therefore we can say that, the $\alpha$-cut and interval arithmetic solution $\bar{X}_{I}$ exists and its components are continuous triangular shaped fuzzy numbers

$$
\bar{x}_{1} \approx\left(-\frac{1}{3} / \frac{1}{2} / 2\right), \bar{x}_{2} \approx\left(\frac{1}{5} / \frac{1}{2} / 1\right) \text { and } \bar{x}_{3} \approx\left(\frac{1}{6} / \frac{5}{8} / \frac{4}{3}\right) .
$$

The membership function of the triangularly shaped number $\bar{x}_{1} \approx\left(-\frac{1}{3} / \frac{1}{2} / 2\right)$ is $x=\frac{(-1+2 \alpha)}{(3-\alpha)} \Rightarrow \mu_{1 L}(x)=\alpha=\frac{(1+3 x)}{(2+x)}$, for $-\frac{1}{3} \leq x \leq \frac{1}{2}$, and

$$
x=\frac{2-\alpha}{1+\alpha} \Rightarrow \mu_{1 U}(x)=\alpha=\frac{(2-x)}{(1+x)}, \text { for } \frac{1}{2} \leq x \leq 2 .
$$

Thus the membership function of $\bar{x}_{1} \approx\left(-\frac{1}{3} / \frac{1}{2} / 2\right)$ is

$$
\mu_{\bar{x}_{1}}(x)= \begin{cases}\frac{(1+3 x)}{(2+x)}, & \text { for }-\frac{1}{3} \leq x \leq \frac{1}{2} \\ \frac{(2-x)}{(1+x)}, & \text { for } \frac{1}{2} \leq x \leq 2 \\ 0, & \text { otherwise }\end{cases}
$$

and its graph is shown in Figure 9.

The membership function of the triangular shaped number $\bar{x}_{2} \approx\left(\frac{1}{5} / \frac{1}{2} / 1\right)$ is

$$
\begin{gathered}
x=\frac{(1+\alpha)}{(5-\alpha)} \Rightarrow \mu_{2 L}(x)=\alpha=\frac{(-1+5 x)}{(1+x)}, \text { for } \frac{1}{5} \leq x \leq \frac{1}{2}, \text { and } \\
x=\frac{(3-\alpha)}{(3+\alpha)} \Rightarrow \mu_{2 U}(x)=\alpha=\frac{(3-3 x)}{(1+x)}, \text { for } \frac{1}{2} \leq x \leq 1 .
\end{gathered}
$$




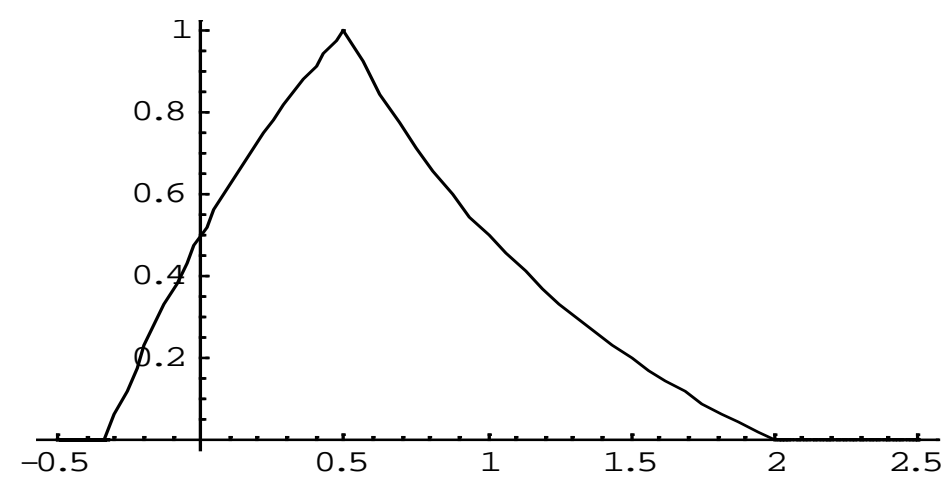

Figure 9. Graph of the membership function of $\bar{x}_{1} \approx\left(-\frac{1}{3} / \frac{1}{2} / 2\right)$.

Thus the membership function of $\bar{x}_{2} \approx\left(\frac{1}{5} / \frac{1}{2} / 1\right)$ is

$$
\mu_{\bar{x}_{2}}(x)= \begin{cases}\frac{(-1+5 x)}{(1+x)}, & \text { for } \frac{1}{5} \leq x \leq \frac{1}{2} \\ \frac{(3-3 x)}{(1+x)}, & \text { for } \frac{1}{2} \leq x \leq 1 \\ 0, & \text { otherwise }\end{cases}
$$

and its graph is shown in Figure 10 .

And the membership function of the triangularly shaped number $\bar{x}_{3} \approx\left(\frac{1}{6} / \frac{5}{8} / \frac{4}{3}\right)$ is $x=\frac{2+3 \alpha}{12-4 \alpha} \Rightarrow \mu_{3 L}(x)=\alpha=\frac{(-2+12 x)}{(3+4 x)}$, for $\frac{1}{6} \leq x \leq \frac{5}{8}$, and $x=\frac{8-3 \alpha}{6+2 \alpha} \Rightarrow \mu_{3 U}(x)=\alpha=\frac{(8-6 x)}{(3+2 x)}$, for $\frac{5}{8} \leq x \leq \frac{4}{3}$.

Thus the membership function of $\bar{x}_{3} \approx\left(\frac{1}{6} / \frac{5}{8} / \frac{4}{3}\right)$ is

$$
\mu_{\bar{x}_{3}}(x)= \begin{cases}\frac{(-2+12 x)}{(3+4 x)}, & \text { for } \frac{1}{6} \leq x \leq \frac{5}{8} \\ \frac{(8-6 x)}{(3+2 x)}, & \text { for } \frac{5}{8} \leq x \leq \frac{4}{3} \\ 0, & \text { otherwise }\end{cases}
$$

and its graph is shown in Figure 11.

Finally, the graph of the $\alpha$-cut and interval arithmetic solution

$$
\bar{X}_{I}=\left[\begin{array}{c}
\bar{x}_{1} \approx\left(-\frac{1}{3} / \frac{1}{2} / 2\right) \\
\bar{x}_{2} \approx\left(\frac{1}{5} / \frac{1}{2} / 1\right) \\
\bar{x}_{3} \approx\left(\frac{1}{6} / \frac{5}{8} / \frac{4}{3}\right)
\end{array}\right]
$$

is shown in Figure 12. 


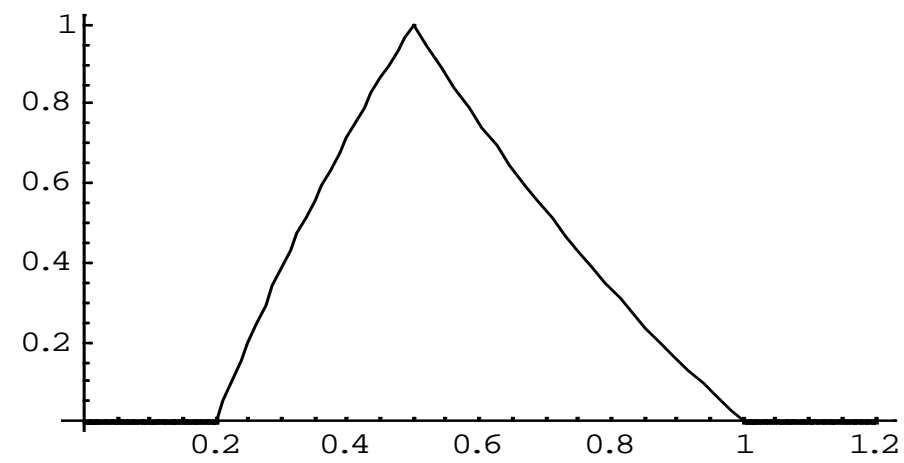

Figure 10. Graph of the membership function of $\bar{x}_{2} \approx\left(\frac{1}{5} / \frac{1}{2} / 1\right)$.

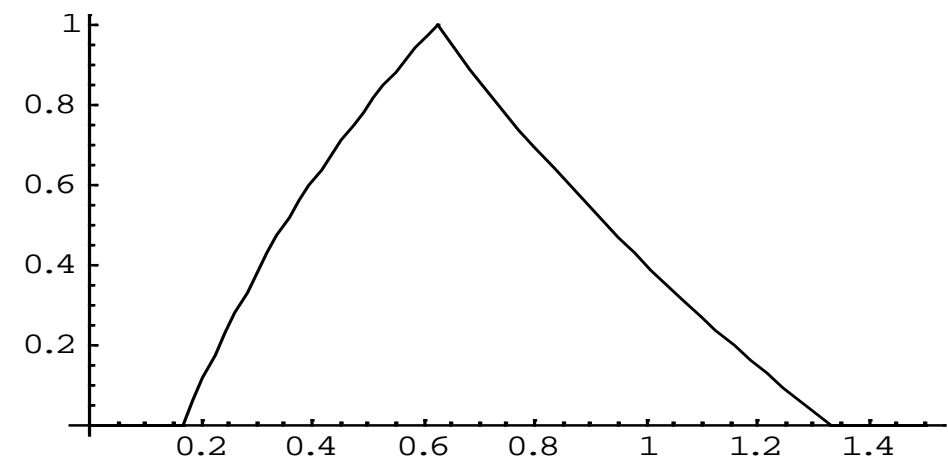

Figure 11. Graph of the membership function of $\bar{x}_{3} \approx\left(\frac{1}{6} / \frac{5}{8} / \frac{4}{3}\right)$.

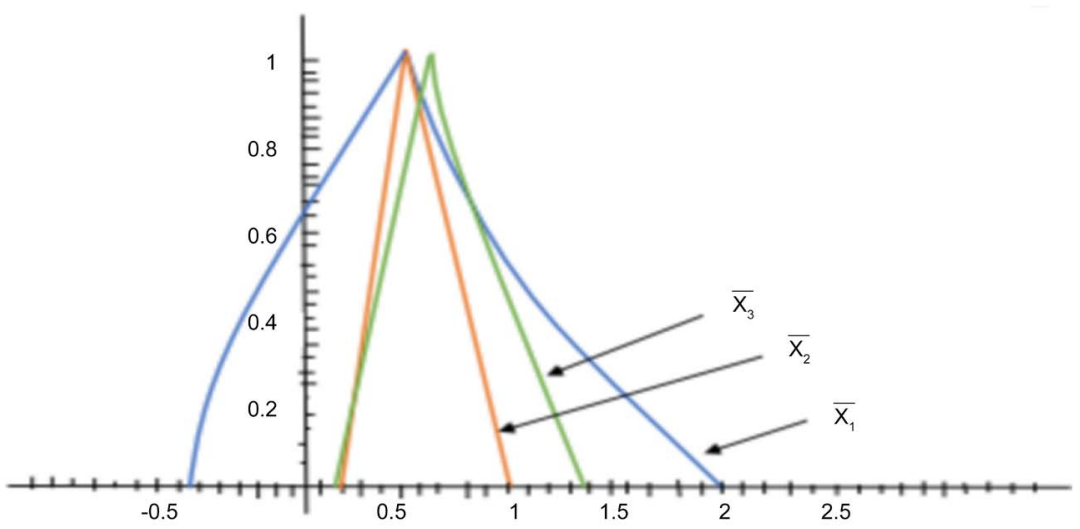

Figure 12. Graph of the $\alpha$-cut and interval arithmetic solution $\bar{X}_{I}$.

\section{Results}

Now we compare the classical solution $\bar{X}_{c}$, extension principle solution $\bar{X}_{e}$, and $\alpha$-cut and interval arithmetic solution $\bar{X}_{I}$ for the system

$$
\left[\begin{array}{ccc}
(1 / 2 / 3) & 0 & 0 \\
0 & (3 / 4 / 5) & 0 \\
0 & 0 & (6 / 8 / 12)
\end{array}\right]\left[\begin{array}{l}
\bar{x}_{1} \\
\bar{x}_{2} \\
\bar{x}_{3}
\end{array}\right]=\left[\begin{array}{c}
(-1 / 1 / 2) \\
(1 / 2 / 3) \\
(2 / 5 / 8)
\end{array}\right]
$$

From the above discussion, we get the solutions as follows 


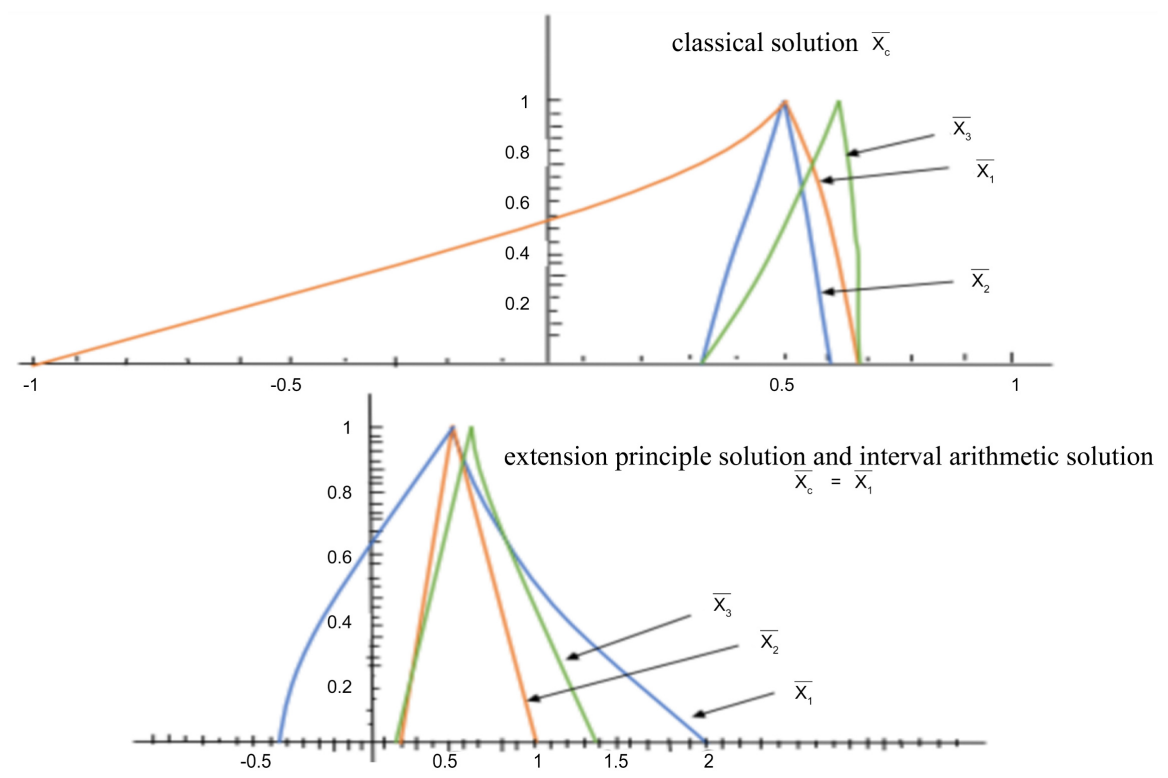

Figure 13. Comparison of the classical solution $\bar{X}_{c}$, extension principle solution $\bar{X}_{e}$, and $\alpha$-cut and interval arithmetic solution $\bar{X}_{e}$.

$\bar{X}_{c}=\left[\begin{array}{l}\bar{X}_{1} \approx\left(-1 / \frac{1}{2} / \frac{2}{3}\right) \\ \bar{x}_{2} \approx\left(\frac{1}{3} / \frac{1}{2} / \frac{3}{5}\right) \\ \bar{x}_{3} \approx\left(\frac{1}{3} / \frac{5}{8} / \frac{2}{3}\right)\end{array}\right], \quad \bar{X}_{e}=\left[\begin{array}{c}\bar{x}_{1} \approx\left(-\frac{1}{3} / \frac{1}{2} / 2\right) \\ \bar{x}_{2} \approx\left(\frac{1}{5} / \frac{1}{2} / 1\right) \\ \bar{x}_{3} \approx\left(\frac{1}{6} / \frac{5}{8} / \frac{4}{3}\right)\end{array}\right]$ and $\bar{X}_{I}=\left[\begin{array}{c}\bar{x}_{1} \approx\left(-\frac{1}{3} / \frac{1}{2} / 2\right) \\ \bar{x}_{2} \approx\left(\frac{1}{5} / \frac{1}{2} / 1\right) \\ \bar{x}_{3} \approx\left(\frac{1}{6} / \frac{5}{8} / \frac{4}{3}\right)\end{array}\right]$.

Here we see that, extension principle solution $\bar{X}_{e}$ and $\alpha$-cut and interval arithmetic solution $\bar{X}_{I}$ are equal. That is, $\bar{X}_{e}=\bar{X}_{I}$. Hence we can say, $\bar{X}_{c} \leq \bar{X}_{e} \leq \bar{X}_{I}$. The comparison among the classical solution $\bar{X}_{c}$, extension principle solution $\bar{X}_{e}$, and $\alpha$-cut and interval arithmetic solution $\bar{X}_{I}$ is shown in Figure 13.

\section{Conclusion}

The system of fuzzy linear equations undoubtedly plays a vital role in presently applied mathematics. Here our intention was to establish some models of solving that system and we presented three different methods of with their applications. We came to know by the above discussion that among the three models extension principle solution $\bar{X}_{e}$, and $\alpha$-cut and interval arithmetic solution $\bar{X}_{I}$ give the same results. In the graphical representation we, find that the extension principle solution $\bar{X}_{e}$, and $\alpha$-cut and interval arithmetic solution $\bar{X}_{I}$ meet at the same point but the classical solution $\bar{X}_{c}$ is deviated a bit from the other two. Actually the solving techniques and their comparison are the ultimate findings of our work.

\section{Conflicts of Interest}

The authors declare no conflicts of interest regarding the publication of this paper. 


\section{References}

[1] Moore, R.E. (1979) Methods and Applications of Interval Analysis. SIAM, Philadelphia. https://doi.org/10.1137/1.9781611970906

[2] Allahviranloo, T., Salahshour, S., Homayoun-nejad, M. and Baleanu, D. (2013) General Solutions of Fully Fuzzy Linear System. General Solutions of Fully Fuzzy Linear System, 2013, Article ID: 593274. https://doi.org/10.1155/2013/593274

[3] Friedman, M., Ma, M. and Kandel, A. (1998) Fuzzy Linear Systems. Fuzzy Sets and Systems, 96, 201-209. https://doi.org/10.1016/S0165-0114(96)00270-9

[4] Allahviranloo, T. (2004) Numerical Methods for Fuzzy System of Linear Equations. Applied Mathematics and Computation, 155, 493-502. https://doi.org/10.1016/S0096-3003(03)00793-8

[5] Allahviranloo, T. (2005) Successive over Relaxation Iterative Method for a Fuzzy System of Linear Equations. Applied Mathematics and Computation, 162, 189-196. https://doi.org/10.1016/j.amc.2003.12.085

[6] Allahviranloo, T. (2005) The Adomian Decomposition Method for a Fuzzy System of Linear Equations. Applied Mathematics and Computation, 163, 553-563. https://doi.org/10.1016/j.amc.2004.02.020

[7] Allahviranloo, T., Ahmady, E., Ahmady, N. and Shams Alketaby, Kh. (2006) Block Jacobi Two-Stage Method with Gauss-Sidel Inner Iterations for Fuzzy System of Linear Equations. Applied Mathematics and Computation, 175, 1217-1228. https://doi.org/10.1016/j.amc.2005.08.047

[8] Dehghan, M., Hashemi, B. and Ghatee, M. (2007) Solution of the Fully Fuzzy Linear Systems Using Iterative Techniques. Chaos Solutions and Fractals, 34, 316-336. https://doi.org/10.1016/j.chaos.2006.03.085

[9] Dehghan, M. and Hashemi, B. (2006) Solution of the Fully Fuzzy Linear Systems Using the Decomposition Procedure. Applied Mathematics and Computation, 182, 1568-1580. https://doi.org/10.1016/j.amc.2006.05.043

[10] Ming, M., Friedman, M. and Kandel, A. (1997) General Fuzzy Least Squares. Fuzzy Sets and Systems, 88, 107-118. https://doi.org/10.1016/S0165-0114(96)00051-6

[11] Klir, G.J. (2013) Lotfi Zadeh, Fuzzy Sets, and I: A Personal Odyssey. In: Seising R., Trillas, E., Moraga, C. and Termini, S., Eds., On Fuzziness. Studies in Fuzziness and Soft Computing, Vol. 298, Springer, Berlin, Heidelberg.

[12] Buckley, J.J. (1992) Solving Fuzzy Equations. Fuzzy Sets and Systems, 50, 1-14. https://doi.org/10.1016/0165-0114(92)90199-E 